\title{
Parallel Synaptic Acetylcholine Signals Facilitate Large Monopolar Cell Repolarization and Modulate Visual Behavior in Drosophila
}

\author{
Jinglin Wu, ${ }^{1}$ Xiaoxiao Ji, ${ }^{1}$ Qiuxiang Gu, ${ }^{1}$ Buxin Liao, ${ }^{1}$ Wei Dong, ${ }^{4}$ and ${ }^{\circledR}$ Junhai Han ${ }^{1,2,3}$ \\ ${ }^{1}$ School of Life Science and Technology, Key Laboratory of Developmental Genes and Human Disease, Southeast University, Nanjing, 210096, \\ China, ${ }^{2}$ Co-innovation Center of Neuroregeneration, Nantong University, Nantong, 226019, China, ${ }^{3}$ Department of Neurology, Affiliated ZhongDa \\ Hospital, Institute of Neuropsychiatry, Southeast University, Nanjing, Jiangsu 210009, China, and ${ }^{4}$ Key Laboratory of Medical Electrophysiology of \\ Ministry of Education and Medical Electrophysiological Key Laboratory of Sichuan Province, Institute of Cardiovascular Research, Southwest \\ Medical University, Luzhou, Sichuan 646000, China
}

Appropriate termination of the photoresponse in image-forming photoreceptors and downstream neurons is critical for an animal to achieve high temporal resolution. Although the cellular and molecular mechanisms of termination in image-forming photoreceptors have been extensively studied in Drosophila, the underlying mechanism of termination in their downstream large monopolar cells remains less explored. Here, we show that synaptic ACh signaling, from both amacrine cells (ACs) and L4 neurons, facilitates the rapid repolarization of L1 and L2 neurons. Intracellular recordings in female flies show that blocking synaptic ACh output from either ACs or L4 neurons leads to slow repolarization of L1 and L2 neurons. Genetic and electrophysiological studies in both male and female flies determine that L2 neurons express ACh receptors and directly receive ACh signaling. Moreover, our results demonstrate that synaptic ACh signaling from both ACs and L4 neurons simultaneously facilitates ERG termination. Finally, visual behavior studies in both male and female flies show that synaptic ACh signaling, from either ACs or L4 neurons to L2 neurons, is essential for the optomotor response of the flies in high-frequency light stimulation. Our study identifies parallel synaptic ACh signaling for repolarization of L1 and L2 neurons and demonstrates that synaptic ACh signaling facilitates L1 and L2 neuron repolarization to maintain the optomotor response of the fly on high-frequency light stimulation.

Key words: ACh; Drosophila; large monopolar cell; synapse; vision; visual behavior

Significance Statement

The image-forming photoreceptor downstream neurons receive multiple synaptic inputs from image-forming photoreceptors and various types of interneurons. It remains largely unknown how these synaptic inputs modulate the neural activity and function of image-forming photoreceptor downstream neurons. We show that parallel synaptic ACh signaling from both amacrine cells and L4 neurons facilitates rapid repolarization of large monopolar cells in Drosophila and maintains the optomotor response of the fly on high-frequency light stimulation. This work is one of the first reports showing how parallel synaptic signaling modulates the activity of large monopolar cells and motion vision simultaneously.

Received Sep. 13, 2020; revised Dec. 3, 2020; accepted Jan. 3, 2021

Author contributions: J.W. and J.H. designed research; J.W., X.J., and Q.G. performed research; J.W., B.L., W.D., and J.H. analyzed data; W.D. edited the paper; J.H. wrote the paper.

This work was supported by National Natural Science Foundation of China Grant 31771171 and Guangdong Key Project 2018B030335001 Grant to J.H. We thank Dr. Jens Rister for L1-GAL4 flies; Dr. Aljoscha Nern for Lai-GAL4, L4-GAL4, and L2-GAL4 flies; Dr. Jianquan Ni for providing RNAi flies; Bloomington Stock Center and Vienna Drosophila RNAi Center for the flies; Dr. Li Liu for providing the platform of motion detection behavior test; Dr. Ying Shen for helping in kinetic fitness; and members of the J.H. laboratory for critical comments on the manuscript.

The authors declare no competing financial interests.

Correspondence should be addressed to Junhai Han at junhaihan@seu.edu.cn or Wei Dong at dongwei@swmu.edu.cn.

https://doi.org/10.1523/JNEUROSCI.2388-20.2021

Copyright $\odot 2021$ the authors

\section{Introduction}

Image-forming vision initiates with light activation of rods and cones in the vertebrate retina or eight photoreceptors (R1-R8) in the Drosophila retina, which send information about the color, contrast, and motion of objects to brain. In addition, Melanopsin in the vertebrate intrinsically photosensitive retinal ganglion cells or the flavoprotein Cryptochrome and Rhodopsin 7 in Drosophila pacemaker neurons generate non-image-forming vision to synchronize the biological clocks with the external light/dark cycle (Berson et al., 2002; Hattar et al., 2002; Ni et al., 2017; Baik et al., 2019). In both vertebrates and invertebrates, absorption of light photons in 
Table 1. The cells targeted by individual fly lines and the original source publications

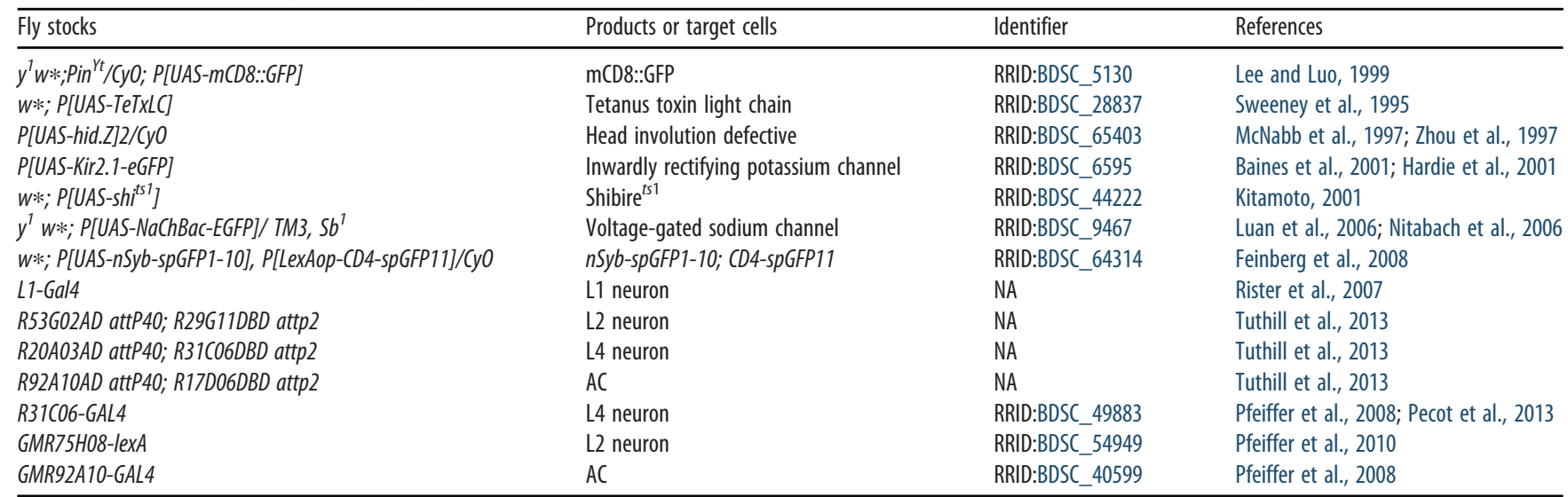

image-forming photoreceptors causes a conformational change of the light receptor rhodopsin, which subsequently activates the phototransduction cascade. The image-forming photoreceptors promptly return to their original state when the light is turned off (Montell, 1999; Burns and Baylor, 2001; Hardie and Raghu, 2001; Tian et al., 2012). Rapid termination of photoresponses is critical for image-forming vision, whereas a sustained photoresponse is essential for non-image-forming vision (Montell, 1999; Berson et al., 2002; Hattar et al., 2002; Han et al., 2007; Ni et al., 2017; Baik et al., 2019). Rapid termination of the image-forming photoreceptor response depends on intrinsic regulation at each step of phototransduction, including the deactivation of rhodopsin as well as the restoration the components of the phototransduction cascade (Laitko and Hofmann, 1998; Burns and Baylor, 2001; Han et al., 2006; Wang and Montell, 2007; Hu et al., 2012). In addition, extrinsic regulation also contributes to the termination of the image-forming photoreceptor response (e.g., retrograde regulation from retinal horizontal cells and visual glial cells) (Rajaram et al., 2005; Bringmann et al., 2006; Cadetti and Thoreson, 2006; Chaturvedi et al., 2014). However, the underlying mechanism for rapid termination of downstream neurons remains less explored.

The Drosophila visual system is a good model to dissect the molecular and neural circuit mechanisms for neural activity modulation. In Drosophila, the compound eye contains $\sim 800$ ommatidia, and each ommatidium contains eight photoreceptors (R1-R8). The outer photoreceptors (R1-R6) project axons from the retina to the underlying lamina neuropil to form synapses with large monopolar cells (LMCs, L1-L3) and amacrine cells (ACs) in lamina, where they are organized into synaptic modules called cartridges (Tian et al., 2012). Light stimulation activates phototransduction cascades in photoreceptors to release the inhibitory neurotransmitter histamine (Hardie, 1989; Tian et al., 2012), which subsequently hyperpolarizes postsynaptic LMCs (L1-L3) and ACs by opening their HisCl2 channels (Huguenard, 1996; Gisselmann et al., 2002; Pantazis et al., 2008; Hu et al., 2015). Conversely, turning the light off abolishes histamine release and excites the postsynaptic neurons. ERG recording is an extracellular recording technique that can measure the lightinduced mass responses of the eye, and $\mathrm{ON}$ and OFF transients in ERG reflect the electrical activity of LMCs at the onset and the end of light stimulation, respectively (Heisenberg, 1971; Agee and Park, 1975). The L1 and L2 neurons are electrically coupled via gap junctions, and serial electron microscopy reconstruction has shown that L1 and L2 neurons receive multiple inputs from
R cells, L4 neurons, ACs, C2, C3, and Lawf neurons (Joesch et al., 2010; Rivera-Alba et al., 2011). However, the mechanism that regulates LMC repolarization remains unknown.

In this study, we show that ACs form synaptic connections with L2 neurons, and L2 neurons directly receive ACh signaling from ACs to facilitate the rapid repolarization of L1 and L2 neurons. Synaptic ACh signaling from L4 neurons also facilitates L1 and L2 neuron repolarization. Moreover, we demonstrate that synaptic ACh signaling from either ACs or L4 neurons to L1 and L2 neurons is essential for the optomotor response of flies in response to high-frequency light stimulation. Our studies elucidate the molecular mechanism and physiological roles of parallel synaptic ACh signaling in L1 and L2 neuron repolarization and in Drosophila visual signal processing.

\section{Materials and Methods}

Fly stocks. UAS-RNAi lines were ordered from Vienna Drosophila RNAi Center, Tsinghua RNAi Stock Center, and Bloomington Stock Center. The split Lai-GAL4 line (R92A10ADattP40; R17D06DBDattp2) labeling AC cells, L4-GAL4 line (R20A03ADattP40; R31C06DBDattp2) labeling L4 neurons, and L2-GAL4 line (R53G02ADattP40; R29G11DBD attp2) labeling L2 neurons were provided by Aljoscha Nern (Tuthill et al., 2013). The L1-GAL4 line that labels L1 neurons was obtained from Jens Rister (Rister et al., 2007). Other lines used in this work were obtained from the Bloomington Stock Center. All fly lines used, the cells targeted by the individual line, and the original source publications of each line are listed in Table 1. The WT flies used in this study were $w^{1118}$. All flies were maintained in standard medium at $25^{\circ} \mathrm{C}$, with $60 \%-80 \%$ relative humidity. Less than 3-d-old flies were used for ERG recordings, and 3-d-old flies were used for optomotor response assays.

Antibodies. The sources of antibodies are as follows: rabbit anti-histamine (ImmunoStar, RRID:AB_572245), rabbit anti-GFP (Invitrogen, RRID: AB_221569), mouse anti-24B10 (DSHB, RRID:SCR_013527), Alexa Fluor488 goat anti-rabbit IgG (Abcam, RRID:AB_2734747), Alexa Fluor-555 goat anti-rabbit IgG (Abcam, catalog \#ab150086), and Alexa Fluor-647 goat anti-mouse IgG (Abcam, RRID:AB_2687948).

Electroretinogram recordings. The ERG recordings were conducted at $25^{\circ} \mathrm{C}$ as previously described (Tian et al., 2013). Flies $<3 \mathrm{~d}$ old were collected and immobilized with strips of tape. Before recording, the flies were adapted to darkness for $5 \mathrm{~min}$. Two borosilicate tubing microelectrodes (World Precision Instruments, RRID:SCR_008593) were used as recording electrodes, and reference electrodes filled with Ringer's solution were placed on the surfaces of the compound eye and shoulder. Fly eyes were stimulated with $5 \mathrm{~s}$ light pulses (4000 Lux). For each genotype and condition, $>8$ flies were examined. ERG recording traces were analyzed using pCLAMp 10.6 software (Molecular Devices, RRID:SCR 011323), and the averaged decay traces of ERG recording were fitted using GraphPad Prism 7 (GraphPad Software, RRID:SCR_002798) to 
corroborate the recordings. To quantitate the contribution of fast decay and the speed of response termination, the time required for $1 / 4$ recovery of amplitude $\left(\mathrm{t}_{1 / 4}\right)$ was measured and the SE was calculated.

Intracellular recordings. Intracellular recordings in photoreceptors were performed as previously described (Hu et al., 2015). A fly was fixed with glue in a fly-shaped hole cut in steel foil. To allow the recording microelectrode to be inserted into the retina/lamina, a small hole (6-10 ommatidia) was cut in the dorsal cornea, and Vaseline was applied to the eye to prevent drying. A low-resistance borosilicate tubing microelectrode filled with $3 \mathrm{M} \mathrm{KCl}$ was placed into a small hole on the compound eye and used for intracellular recordings from R1-R6 photoreceptors. A reference electrode was filled with Ringer's solution and placed at the retina layer. The recording microelectrode placed on the hole was gradually inserted into the opening until light-induced membrane depolarization was observed. The signals were amplified and recorded using a Warner IE210 Intracellular Electrometer.

Intracellular recordings in LMCs were performed as previously described (L. Zheng et al., 2006). A high-resistance quartz microelectrode (Sutter Instruments, catalog \#QF100-50-10), filled with $3 \mathrm{M}$ potassium acetate with $0.5 \mathrm{mM} \mathrm{KCl}$ (to maintain the chloride battery homeostasis) was used for intracellular recordings for LMCs. Threeday-old adult female flies were used in the experiments; the female flies are larger than the males, making the recordings somewhat easier. A reference electrode, filled with Ringer's solution, was gently pushed through ocelli $\sim 100 \mu \mathrm{m}$ into the head for in vivo intracellular recordings. The recording microelectrode placed on the hole was gradually inserted into the opening until light-induced membrane hyperpolarization was observed. The signals were amplified and recorded using a Warner IE210 Intracellular Electrometer. Intracellular recording traces were analyzed using pCLAMp 10.6 software (Molecular Devices, RRID:SCR_011323), and individual traces of the decay in intracellular recording were fitted using GraphPad Prism 7 to corroborate the recordings.

Histamine staining. Histamine staining was performed as previously described (Chaturvedi et al., 2014). Briefly, fly heads were fixed with $4 \%$ ethyl-dimethyl-aminopropyl-carbodiimide in 0.1 M PBS, pH 7.4, for $1 \mathrm{~h}$ on ice, and then fixed with $4 \%$ PFA in $0.1 \mathrm{M} \mathrm{PBS}, \mathrm{pH} 7.4$, for $1 \mathrm{~h}$ on ice. After three PBS washes, fixed heads were immersed in $12 \%$ glucose with PBS overnight at $4^{\circ} \mathrm{C}$ embedded in optimal cutting temperature medium, and $10-\mu \mathrm{m}$-thick cryosections were immunolabeled with mouse anti-24B10 and rabbit anti-histamine (1:100, ImmunoStar) as primary antibodies. For histamine immunolabeling, the antibody was preadsorbed with carcinine. After three washes with PBS, sections were incubated at room temperature for $2 \mathrm{~h}$ with secondary antibodies Alexa Fluor-555 goat anti-rabbit IgG (1:200, Abcam) and Alexa Fluor-647 goat anti-mouse IgG (1:100, Abcam). After three washes with PBS, brains were mounted for microscopy in Vectashield without DAPI (Vector Laboratories, RRID:SCR_000821), and the images were recorded with an LSM 700 confocal microscope (Carl Zeiss, RRID:SCR_017377).

GFP reconstitution across synaptic partners (GRASP). The neuronalsynaptobrevin::spGFP1-10 chimera (nSyb-spGFP1-10) was used for synaptic GRASP (Macpherson et al., 2015). The UAS-nSyb-spGFP1-10 was expressed in ACs using a Lai-GAL4 driver. The lexAop-CD4-spGFP11 was expressed in L2 neurons under the control of L2-LexA. The reconstituted GFP signals were detected by the anti-GFP antibodies that recognize the reconstituted GFP specifically (1:100; Invitrogen) (Gordon and Scott, 2009). For the control flies, only spGFP11 was expressed in L2 neurons. The axons of photoreceptors in the lamina were labeled with 24B10.

Optomotor responses assay. Fly optomotor responses were tested as previously described (Hu et al., 2015). Briefly, 3-d-old flies were collected, and their wings were cut off. After recovering for $>24 \mathrm{~h}$ in a $12 \mathrm{~h}$ light $/ 12 \mathrm{~h}$ dark cycle incubator, a single fly was placed on a circular platform for the optomotor response detection. The platform was surrounded with a water-filled moat to prevent the fly from escaping, and the moat was surrounded with a panoramic LED display that controlled by LED Studio software (Shenzhen Sinorad Medical Electronics). Bright and dark stripes were used to generate a clockwise motion light for $90 \mathrm{~s}$ followed by a counterclockwise motion light for another 90 s. A $22.5^{\circ}$ spatial wavelength light and dark grating pattern was presented with $100 \%$ contrast values, and three angular velocities (90, 225, and 562.5/s) corresponded to the light stimulation frequencies of 4,10 , and $25 \mathrm{~Hz}$, respectively. The light intensity of the LED screen was 160 Lux. The walking traces of flies were recorded with a camera (WV-BP330, Panasonic System Networks). The coordinates of the position of the fly in each recorded frame were calculated using Limelight software (Coulbourn Instruments, RRID:SCR_014254), and the data were analyzed in MATLAB (The MathWorks, RRID:SCR_010457). The optomotor responses of the flies were quantified by the performance index of tracking time (PITT). The PITT is defined as follows: (tracking time untracking time) $/($ tracking time + untracking time). The probabilities of fly movement in the platform in accordance with LED rotating direction or not are defined as tracking or untracking, respectively.

Experimental design and statistical analysis. For ERG recordings, $>8$ flies were examined, and the fly number used for each genotype and condition is presented in the figures. Two-tailed Student's $t$ tests were used for comparing the quarter of decay time in ERG.

For intracellular recordings, $>4$ flies were examined for each genotype and condition, and only one neuron was recorded from 1 fly. The neuron number used to generate the averaged decay trace is presented in the text. The decay traces of ERG and intracellular recording were fitted using GraphPad Prism 7 to corroborate the recordings. The comparison of reduced $R^{2}$ between single exponential decay and double exponential decay fittings revealed a better fit (higher $R^{2}$ ). $R^{2}$ is informed in the figure legends. Two-tailed Student's $t$ tests were used for comparing the decay constants.

To compare the distribution of histamine in the eye, 4 WT and 7 Lai-Gal4/UAS-TeTxLC flies were examined. Two-tailed Student's $t$ tests were used for comparing the relative histamine signal of lamina over retina.

For fly optomotor responses, $>8$ flies were examined for each genotype and condition. The fly numbers used for optomotor response analysis are presented in the figures. Unpaired $t$ tests were used for comparing the performance index of tracking time.

Statistical analysis was conducted using GraphPad Prism 5 (RRID: SCR_002798). Boxplots were also generated using GraphPad Prism 5. All data are presented as boxplots, box dimension indicates the middle $50 \%$ of the data, and line and whiskers indicate variability outside the upper and lower quartiles. Dots indicate outliers.

\section{Results}

\section{Blocking synaptic ACh release from ACs leads to slow ERG termination}

ERG recordings show that light stimulation evokes a rapid depolarization whereas light cessation results in a rapid repolarization in WT flies (Fig. $1 A$ ). The decay of ERG recording in WT flies is nicely fit with a double exponential decay fit with $\tau_{\text {fast }}=77.73 \mathrm{~ms}, \tau_{\text {slow }}=7803 \mathrm{~ms}$ (Fig. $1 B$ ), suggesting that variant mechanisms regulate ERG termination. Given that the intrinsic regulations for ERG termination of image-forming photoreceptor have been extensively studied, we further investigate the potential roles of neural circuits in ERG termination. Interestingly, blocking all neurotransmitters release from $\mathrm{AC}$ cells via ectopic expression of tetanus toxin light chain (TeTxLC) (Sweeney et al., 1995) results in a different shape of ERG termination. Satisfied single exponential decay fit with $\tau=$ $6686 \mathrm{~ms}$ is obtained for the decay of ERG recording in LaiGal4/UAS-TeTxLC flies (Fig. 1B), indicating that the regulation for the fast decay of ERG recording is disrupted in these flies. These results indicate that the synaptic output from ACs is essential for the fast decay in ERG recording. Therefore, from here on, the time required for a quarter recovery from the ERG responses is used to quantify the contribution of fast decay and the termination speed of ERG recording in each genotype and 

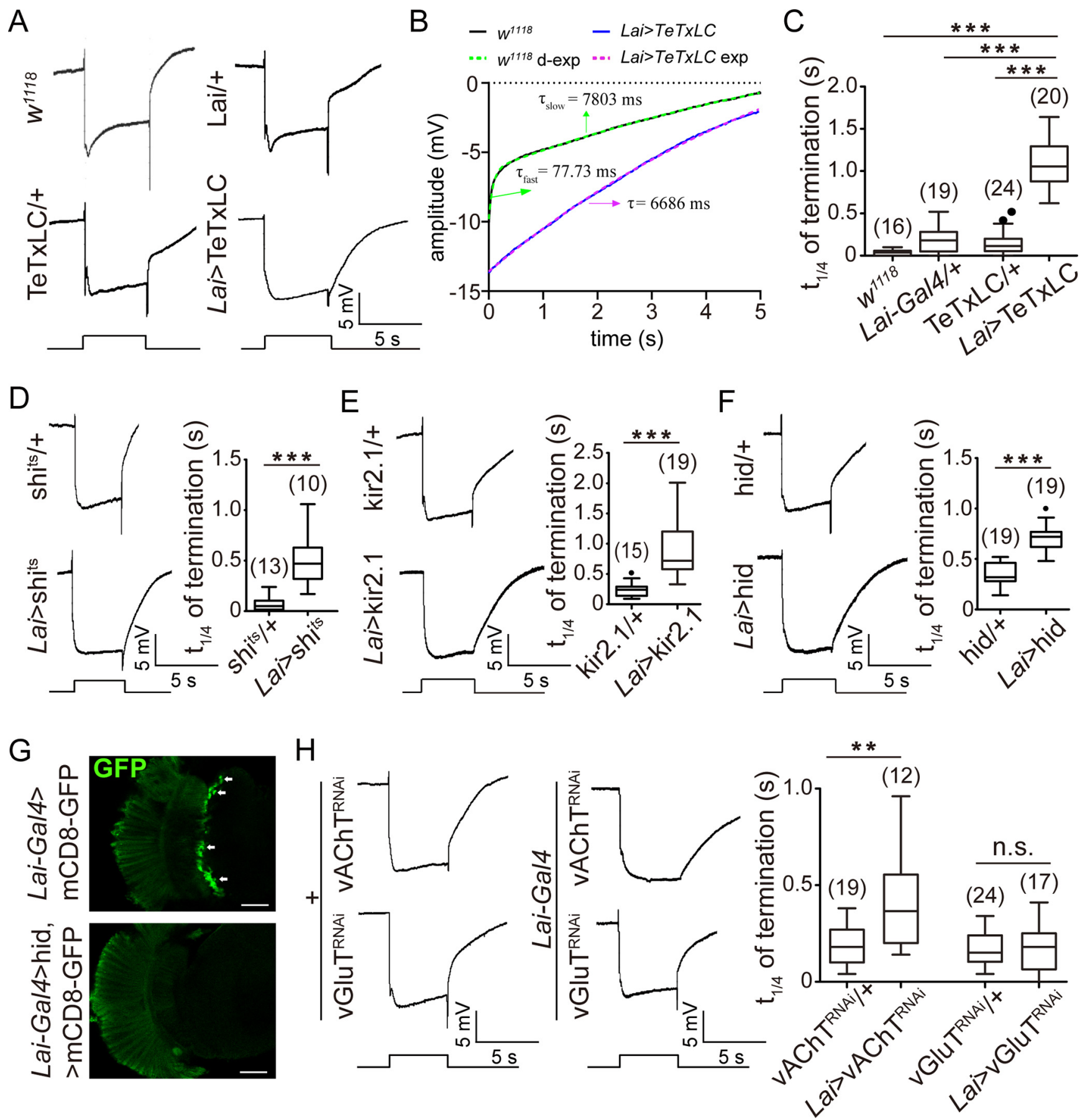

Figure 1. Blocking synaptic ACh release from ACs leads to slow termination in ERG. $\boldsymbol{A}$, ERG recording traces of WT, and flies with expression of TeTxLC using Lai-GAL4 driver. Event markers represent $5 \mathrm{~s}$ orange light pulses. Calibration: ERG traces throughout all figures, $5 \mathrm{mV}$. B. The decay of ERG recordings in WT and Lai-Gal4/UAS-TeTXLC flies is fit with double or single exponential decay fit, respectively. Solid lines indicate the averaged experimental ERG recording decay traces. Dashed lines indicate the exponential decay fit. $w^{1718}: R^{2}=99.72 \% ;$ Lai-Gal4/UAS-TeTxLC: $R^{2}=99.83 \%$. Arrows indicate decay constants for the fast and slow component of the exponential fit. $C$, Boxplots with whiskers represent the times ( $\left.t_{1 / 4}\right)$ required for a $1 / 4$ recovery from the responses on stimulation cessation (with maximum 1.5 interquartile ranges). The number of recorded flies for each genotype are also listed. $\boldsymbol{D}$, ERG recording traces of the flies with expression of shibire ${ }^{\text {ts }}$ using a Lai-GAL4 driver. For this experiment, the flies were kept for at $30.5^{\circ} \mathrm{C}$ for $3 \mathrm{~h}$ before the experiment, and the ERG recordings were conducted at $30.5^{\circ} \mathrm{C}$. Right, Quantification of the time $\left(\mathrm{t}_{1 / 4}\right)$ required for a $1 / 4$ recovery and the number of recorded flies. $E$, ERG recording traces of flies with expression of Kir2.1 using Lai-GAL4 driver. Right, Quantification of the time $\left(t_{1 / 4}\right)$ required for a $1 / 4$ recovery and the number of recorded flies. $\boldsymbol{F}$, ERG recording traces of flies with expression of HID using Lai-GAL4 driver. Right, Quantification of the time ( $\left.t_{1 / 4}\right)$ required for a $1 / 4$ recovery and the number of recorded flies. $G$, Immunostaining images of flies with expression of both mCD8-GFP and HID using Lai-GAL4 driver. White arrows point out the somata of AC cells. Scale bar, $20 \mu \mathrm{m}$. $\boldsymbol{H}$, ERG recording traces of the flies expressing UAS-vGluT-RNAi or UAS-vAChT-RNAi using Lai-GAL4 driver. Right, Quantification of the time ( $\left.\mathrm{t}_{1 / 4}\right)$ required for a $1 / 4$ recovery and the number of recorded flies. $* * p<0.01$. $* * * p<0.001$; n.s., Not significant $(p>0.05)$.

condition. Convincingly, blocking all neurotransmitters release from AC cells via ectopic expression of either TeTxLC or shibire ${ }^{t s}\left(s h i^{t s}\right)$ (Kitamoto, 2001) results in slow ERG termination $\left(w^{1118}: \mathrm{t}_{1 / 4}=\right.$ $0.039 \pm 0.0085$ s, vs Lai-Gal4/UAS-TeTxLC: $\mathrm{t}_{1 / 4}=1.08 \pm 0.064 \mathrm{~s}$, $t_{(34)}=14.47, p<0.001 ;$ Lai-Gal4/+: $\mathrm{t}_{1 / 4}=0.17 \pm 0.031 \mathrm{~s}$, vs LaiGal4/UAS-TeTxLC: $\mathrm{t}_{1 / 4}=1.08 \pm 0.064 \mathrm{~s}, t_{(37)}=12.53, p<0.001$; UAS-TeTxLC/+: $\mathrm{t}_{1 / 4}=0.16 \pm 0.028 \mathrm{~s}$, vs Lai-Gal4/UAS-TeTxLC: $\mathrm{t}_{1 / 4}=1.08 \pm 0.064 \mathrm{~s}, t_{(42)}=13.98, p<0.001 ; U A S-s h i^{t s} /+: \mathrm{t}_{1 / 4}=$ 
A

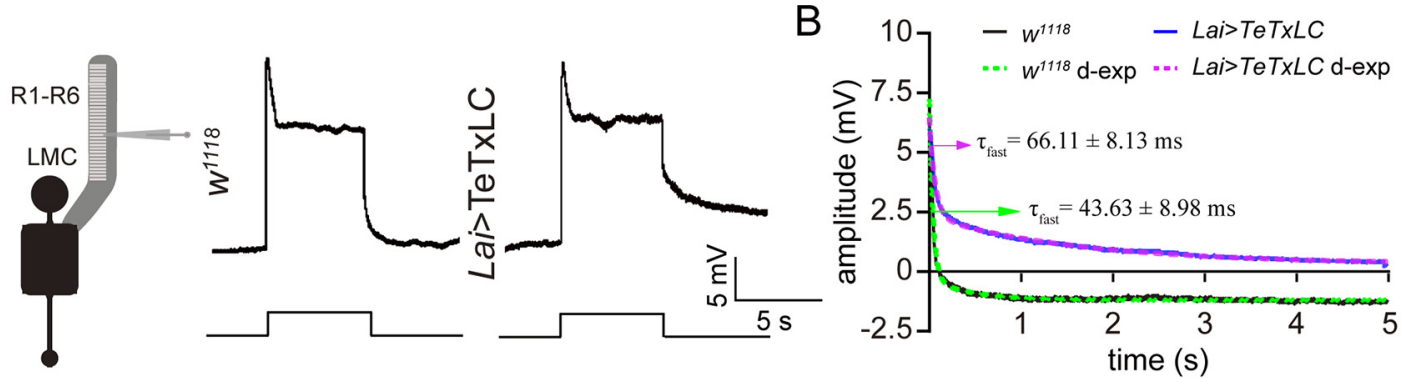

C

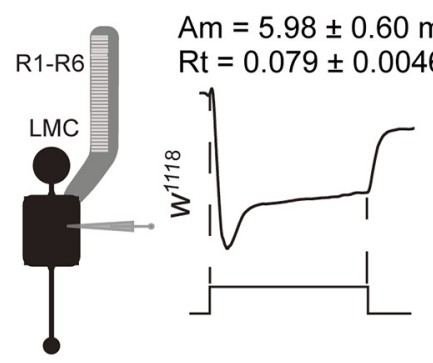

$\mathrm{Am}=6.14 \pm 0.51 \mathrm{mV}$

\author{
D
}

$\mathrm{D}$
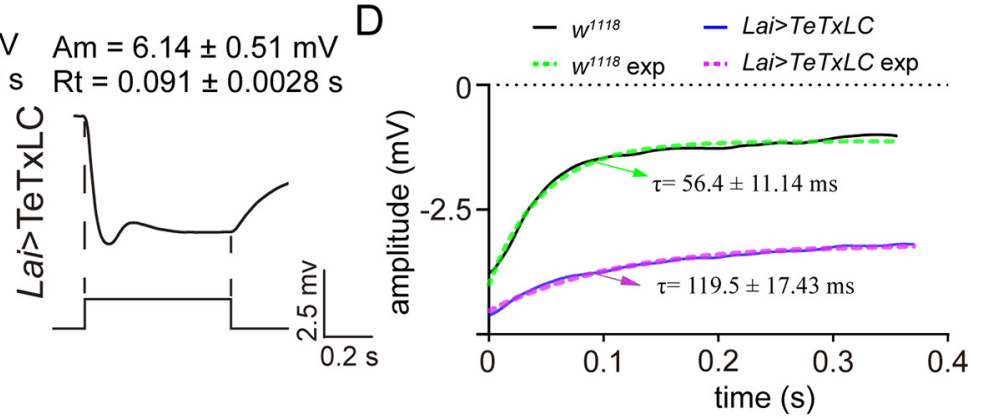

E
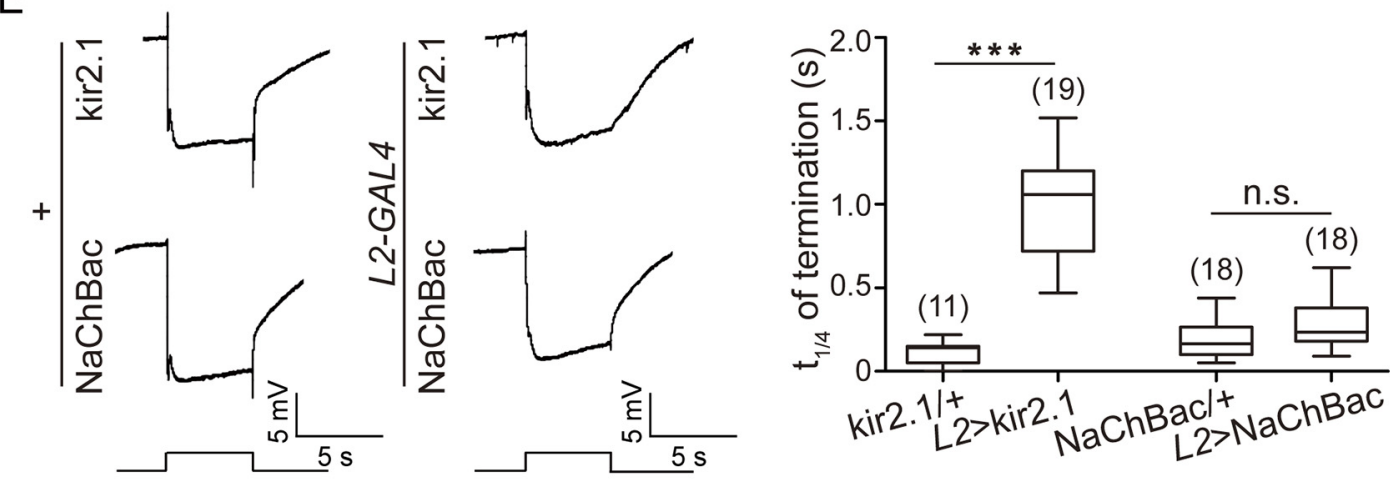

$\mathrm{F}$
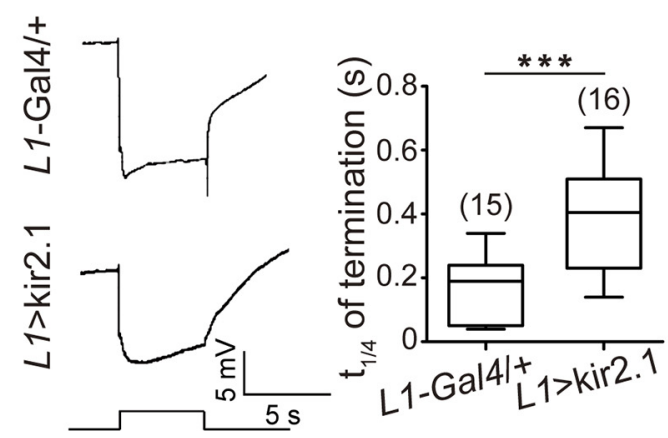

G
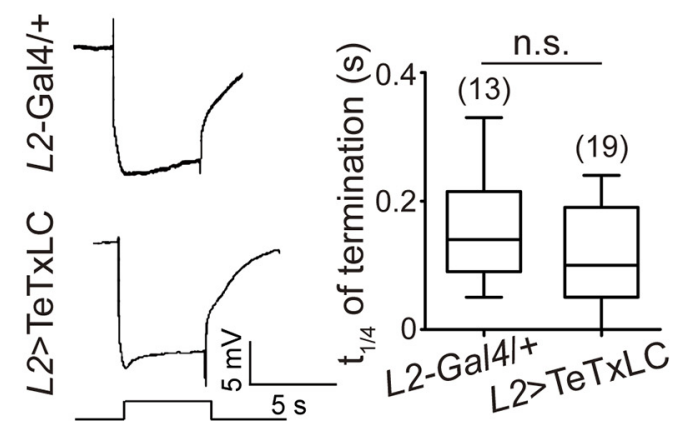

Figure 2. Blocking synaptic ACh release from ACs leads to slow repolarization in L1 and L2 neurons. A, Intracellular recording reveals normal light response in $w^{1118}$ and Lai-GAL4/UASTeTXLC photoreceptors. Left, Simplified diagram of the electrode approach to photoreceptors. Right, Intracellular recording traces. For intracellular recording traces, event markers represent $5 \mathrm{~s}$ orange light pulses. Calibration: $5 \mathrm{mV}$. B. The decay of intracellular recordings in photoreceptors is fit with double exponential decay fit. Solid lines indicate the averaged decay trace of experimental intracellular recording. Dashed lines indicate the exponential decay fit. $w^{1718}: R^{2}=93.37 \%$; Lai-Gal4/UAS-TeTxLC: $R^{2}=98.60 \%$. Arrows indicate decay constants for the fast component of the exponential fit. $C$, Intracellular recording reveals slow repolarization in Lai-GAL4/UAS-TeTXLC LMCs. Left, Simplified diagram of electrode approach to LMCS. Right, Intracellular recording traces. For intracellular recording traces, event markers represent $200 \mathrm{~ms}$ light pulses. Calibration: $2.5 \mathrm{mV}$. Top, Measurements of the amplitude (Am) and rise time (Rt) of light-induced hyperpolarization. $\boldsymbol{D}$, The decay of intracellular recordings in LMCs is fit with the single exponential decay fit. Solid lines indicate the averaged decay trace of experimental intracellular recording. Dashed lines indicate the single exponential decay fit. $w^{1718}: R^{2}=98.90 \%$; Lai-Gal4/UAS-TeTXLC: $R^{2}=97.96 \%$. Arrows indicate decay constants for the exponential fit. $E$, ERG recording traces of L2-GAL4/UAS-kir2.1 and L2-GAL4/UAS-NaChBac flies. Right, Quantification of the time $\left(\mathrm{t}_{1 / 4}\right)$ required for a 1/4 recovery and the number of recorded flies. $\boldsymbol{F}$, ERG recording traces of L1-GAL4/ UAS-kir2.1 and L1-GAL4/+ flies. Right, Quantification of the time $\left(\mathrm{t}_{1 / 4}\right)$ required for a 1/4 recovery and the number of recorded flies. G, ERG recording traces of L2-GAL4/UAS-TeTXLC and L2GAL4/+ flies. Right, Quantification of the time $\left(\mathrm{t}_{1 / 4}\right)$ required for a $1 / 4$ recovery and the number of recorded flies. $* * * p<0.001 ; n$.s., Not significant $(p>0.05)$.

$0.073 \pm 0.019$ s, vs Lai-Gal4/UAS-shits: $\mathrm{t}_{1 / 4}=0.52 \pm 0.086 \mathrm{~s}$, $t_{(21)}=5.73, p<0.001$, two-tailed Student's $t$ test; Fig. $\left.1 C, D\right)$. Similar deficits are observed in the flies with electrically silenced ACs via ectopic expression of the inward rectifier potassium channel (Kir2.1) (Baines et al., 2001) to induce irreversible hyperpolarization (UAS-Kir2.1/+: $\mathrm{t}_{1 / 4}=0.25 \pm 0.03 \mathrm{~s}$, vs Lai-Gal4/UASKir2.1: $\mathrm{t}_{1 / 4}=0.9 \pm 0.095 \mathrm{~s}, t_{(32)}=5.93, p<0.001$, two-tailed Student's $t$ test; Fig. 1E). Similarly, abolishing ACs via 
A

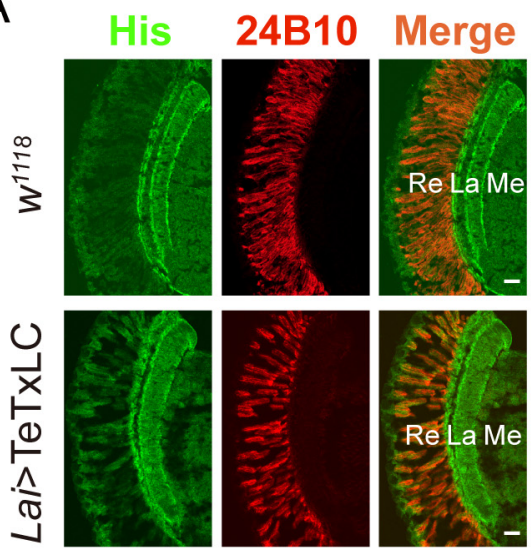

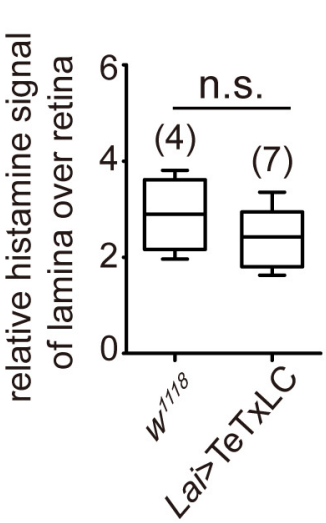

B

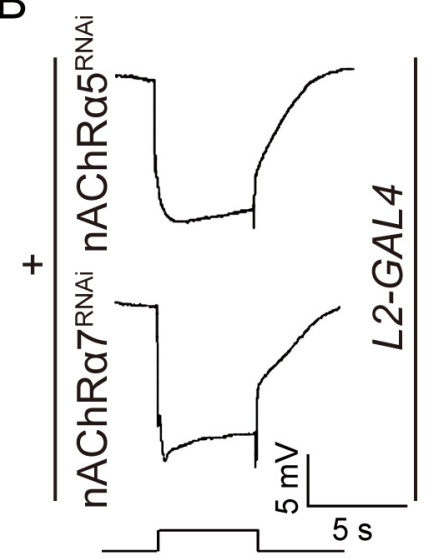

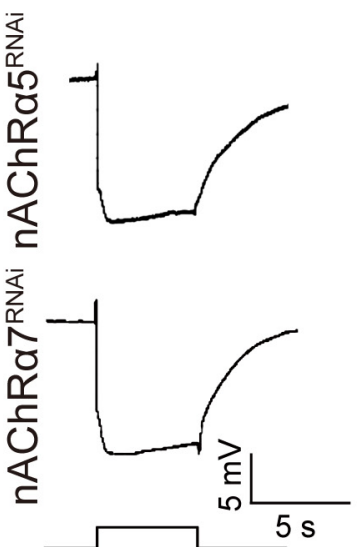

C

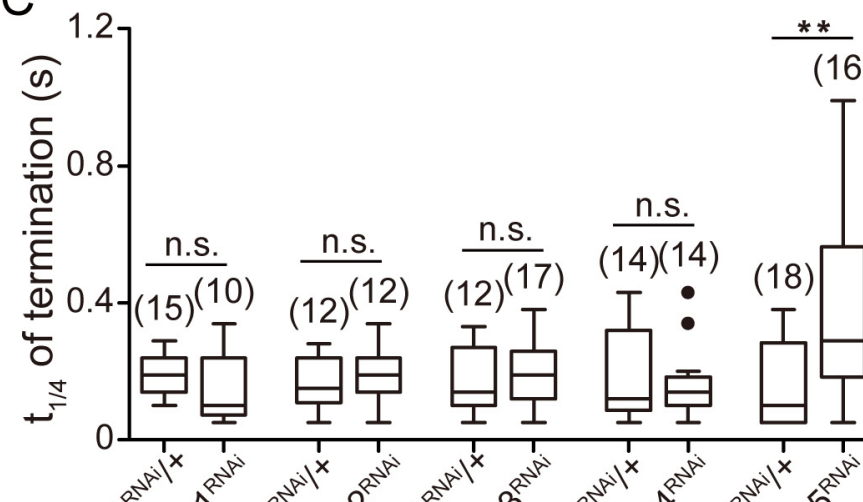

(16)

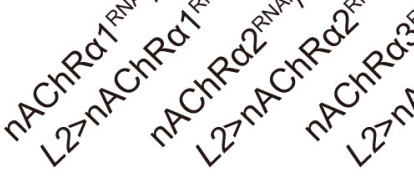

D
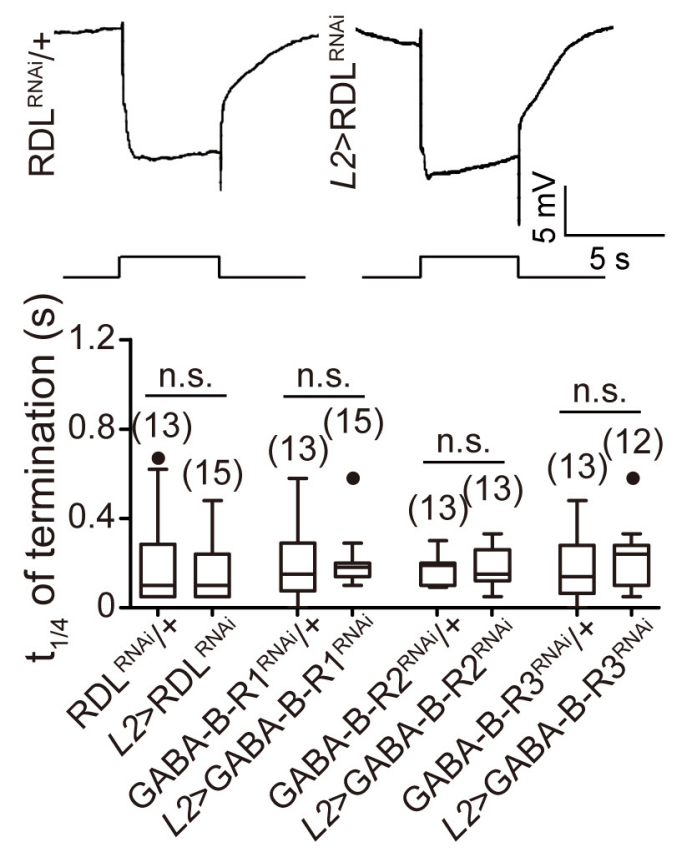

E

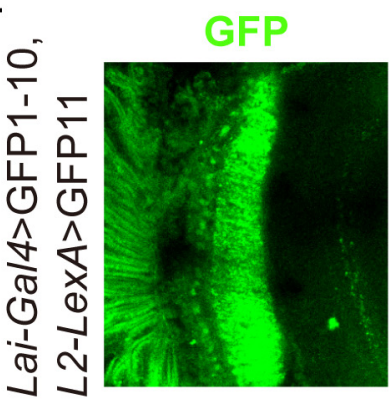

24B10

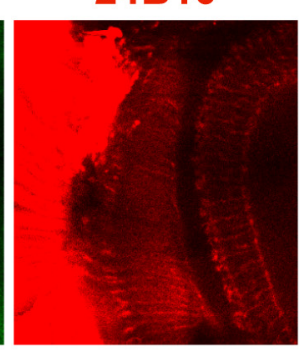

Merge

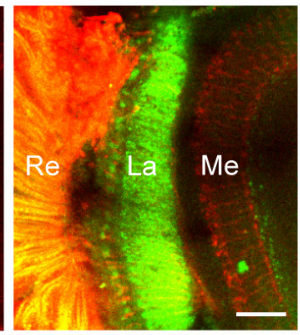

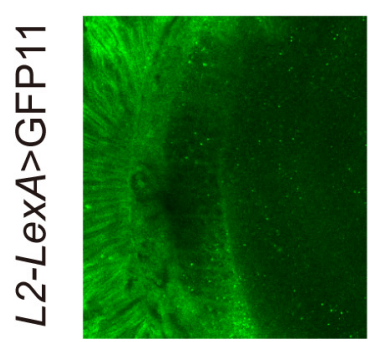
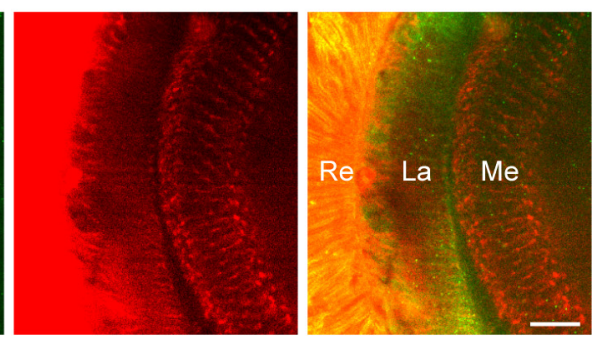

Figure 3. AChRs in $L 2$ neurons receive synaptic ACh release from ACs to facilitate LMCs repolarization. $A$, Immunostaining images represent normal level and distribution of histamine in $w^{1118}$ and Lai-GAL4/UAS-TeTxLC flies. Histamine (green) was immunolabeled in horizontal sections of heads from $w^{1118}$, and Lai-GAL4/UAS-TeTxLC flies were stained with 24B10 (red, for Chaoptin) and antihistamine antibody (green). The anti-24B10 signal is condensed in the retinal (Re), and is weak in the lamina (La) and the medulla (Me). The weak anti-24B10 signal is hard to be detected, when nonsaturated anti-24B10 signal in the retina is observed. Scale bar, $20 \mu \mathrm{m}$. Right, Quantification of relative histamine level and the number of examined flies. $\boldsymbol{B}$, ERG recording traces of L2-GAL4/UAS-nAChR $\alpha 5^{R N A i}$, L2-GAL4/UAS-nAChR $\alpha 7^{R N A i}$, and control flies. C, Quantification of the time ( $\left.\mathrm{t}_{1 / 4}\right)$ required for a $1 / 4$ recovery and the number of recorded flies for 
ectopic expression of a pro-cell death gene (UAS-hid) (McNabb et al., 1997) also causes slow ERG termination (UAS-hid/+: $\mathrm{t}_{1 / 4}=0.34 \pm 0.025$ s, vs Lai-Gal4/UAS-hid: $\mathrm{t}_{1 / 4}=$ $0.7 \pm 0.029 \mathrm{~s}, t_{(36)}=9.63, p<0.001$, two-tailed Student's $t$ test; Fig. $1 F, G)$. These data indicate that blocking the synaptic output of ACs leads to slow ERG termination.

Previous immunocytochemistry studies have shown that ACs are both glutamatergic and cholinergic neurons (Kolodziejczyk et al., 2008). We next examine which neurotransmitter released from ACs contributes to rapid ERG termination. vGluT and vAChT are critical for synaptic output via loading glutamate or ACh into synaptic vesicles, respectively. Interestingly, AC-specific knockdown of vAChT, but not vGluT, phenocopies the slow ERG termination phenotype (UAS-vAChT $T^{R N A i} /+: \mathrm{t}_{1 / 4}=$ $0.17 \pm 0.020 \mathrm{~s}$, vs Lai-Gal4/UAS-vAChT ${ }^{R N A i}: \mathrm{t}_{1 / 4}=0.4 \pm 0.069 \mathrm{~s}$, $t_{(29)}=3.64, p=0.001 ; U A S-v G l u T^{R N A i} /+: \mathrm{t}_{1 / 4}=0.17 \pm 0.016 \mathrm{~s}$, vs Lai-Gal4/UAS- $v$ GluT ${ }^{R N A i}: \mathrm{t}_{1 / 4}=0.18 \pm 0.028 \mathrm{~s}, t_{(39)}=0.21$, $p=0.83$, two-tailed Student's $t$ test; Fig. $1 H$ ). These data show that blocking synaptic ACh release from ACs leads to slow ERG termination.

\section{Blocking synaptic output from ACs results in slow repolarization of $\mathrm{L} 1$ and $\mathrm{L} 2$ neurons}

Given that an ERG is an extracellular record that reflects lightinduced mass response of the eye, we next conducted intracellular recordings in photoreceptors to examine whether slow ERG termination is because of prolonged repolarization of photoreceptors. Our previous studies have identified the retrograde glutamate signaling from ACs to photoreceptor cells facilitates the repolarization of photoreceptor cells (Hu et al., 2015). Consistent with previous observations ( $\mathrm{Hu}$ et al., 2015), blocking synaptic glutamate release from ACs suppresses the recording recovery to the prestimulation baseline (Fig. $2 A$ ). Satisfied double exponential decay fits are obtained for the decay of intracellular recordings in both WT and Lai-Gal4/UAS-TeTxLC fly photoreceptors (Fig. 2B). The fast decay constant of Lai-Gal4/UAS-TeTxLC photoreceptors is comparable with that of WT photoreceptors $\left(w^{1118}: \tau_{\text {fast }}=43.63 \pm 8.98 \mathrm{~ms}\right.$, vs Lai-Gal4/UAS-TeTxLC: $\tau_{\text {fast }}=$ $66.11 \pm 8.13 \mathrm{~ms}, t_{(4)}=1.86, p=0.14$, two-tailed Student's $t$ test; Fig. $2 B$ ). These results reveal that slow ERG termination in LaiGal4/UAS-TeTxLC flies is not because of an abnormality in photoreceptors.

Light stimulation activates the phototransduction cascade in eye photoreceptors to release the inhibitory neurotransmitter histamine, which subsequently hyperpolarizes postsynaptic L1-L3 neurons and ACs by opening their HisCl2 channels (Huguenard, 1996; Pantazis et al., 2008; Tian et al., 2012). We further performed intracellular recordings on L1 and L2 neurons to test whether blocking synaptic output from ACs affects light responses of these neurons. In WT L1 and L2 neurons, light stimulation induces hyperpolarization, whereas turning off the light results in rapid repolarization (Fig. 2C). Satisfied single exponential decay fit with $\tau=56.4 \pm 11.14 \mathrm{~ms}$ is obtained for the decay of intracellular recordings in WT L1

\section{$\leftarrow$}

each genotype. $\boldsymbol{D}$, ERG recording traces of $L 2-G A L 4 / U A S-R D L^{R N A i}$ and $U A S-R D L^{R N A i} /+$ flies. Bottom, Quantification of the time $\left(\mathrm{t}_{1 / 4}\right)$ required for a $1 / 4$ recovery and the number of recorded flies for each genotype. E, GRASP between ACs and L2 neurons. Lai-Gal4::UAS-nSybspGFP1-10 and L2-LeXA.:LexAop-CD4:spGFP11 flies were used to express split GFP1-10 in ACs and split GFP11 in L2 neurons, respectively. When these fly lines were crossed, a reconstituted GFP signal (green) was detected only at the lamina. Re, Retina; La, lamina; Me, medulla. Scale bar, $20 \mu \mathrm{m}$. $* * p<0.01$. n.s., Not significant $(p>0.05)$. and L2 neurons (Fig. 2D). In contrast, in the L1 and L2 neurons of Lai-Gal4/UAS-TeTxLC flies, light stimulation induces normal hyperpolarization and light cessation slows repolarization $\left(w^{1118}\right.$ : single exponential decay fit, $\tau=56.4 \pm 11.14 \mathrm{~ms}$, vs Lai-Gal4/UAS-TeTxLC: single exponential decay fit, $\tau=$ $119.5 \pm 17.43 \mathrm{~ms}, t_{(4)}=3.05, p=0.038$, two-tailed Student's $t$ test) (Fig. 2C,D). These data show that blocking synaptic output from ACs results in slow repolarization of L1 and L2 neurons.

Next, we ask whether slow repolarization of L2 and L1 neurons directly contributes to slow ERG termination. We express Kir2.1 channel in L2 neurons to induce irreversible hyperpolarization and find that L2-GAL4/UAS-Kir2.1 flies exhibit slow ERG termination (UAS-Kir2.1/+: $\mathrm{t}_{1 / 4}=0.16 \pm 0.019 \mathrm{~s}$, vs L2-Gal4/ UAS-Kir2.1: $\mathrm{t}_{1 / 4}=0.97 \pm 0.068 \mathrm{~s}, t_{(28)}=9.39, p<0.001$, twotailed Student's $t$ test; Fig. $2 E$ ), similar to that in Lai-GAL4/UASTeTxLC flies. In contrast, ectopic expression of the $\mathrm{NaChBac}$ channel (Luan et al., 2006; Nitabach et al., 2006) in L2 neurons shows normal ERG termination (UAS-NaChBac/+: $t_{1 / 4}=$ $0.19 \pm 0.028 \mathrm{~s}$, vs L2-Gal4/UAS-NaChBac: $\mathrm{t}_{1 / 4}=0.27 \pm 0.031 \mathrm{~s}$, $t_{(34)}=1.83, p=0.076$, two-tailed Student's $t$ test; Fig. $2 E$ ). Given that L1 and L2 neurons are electrically coupled via gap junctions (Joesch et al., 2010), we examined whether inducing hyperpolarization in L1 neurons also results in slow ERG termination. As expected, expression of Kir2.1 channel in L1 neurons also results in slow ERG termination ( $L 1-$ Gal4/+: $\mathrm{t}_{1 / 4}=0.17 \pm 0.028 \mathrm{~s}$, vs L1-Gal4/UAS-Kir2.1: $\mathrm{t}_{1 / 4}=0.38 \pm 0.039 \mathrm{~s}, t_{(29)}=4.28, p=0.002$, two-tailed Student's $t$ test; Fig. $2 F$ ). These data indicate that slow repolarization of $\mathrm{L} 1$ and $\mathrm{L} 2$ neurons leads to slow ERG termination.

We also exclude the possibility that the abnormalities in the neurons downstream from L2 neurons cause slow ERG termination because blocking the neurotransmitter release from L2 neurons results in normal ERG termination (L2-Gal4/+: $t_{1 / 4}=$ $0.16 \pm 0.024 \mathrm{~s}$, vs L2-Gal4/UAS-TeTxLC: $\mathrm{t}_{1 / 4}=0.11 \pm 0.018 \mathrm{~s}$, $t_{(30)}=1.56, p=0.13$, two-tailed Student's $t$ test; Fig. $\left.2 G\right)$. These results demonstrate that slow repolarization of LMCs causes slow ERG termination in Lai-GAL4/UAS-TeTxLC flies.

\section{L2 neurons receive synaptic ACh signaling from ACs to facilitate light off-induced repolarization}

Since HisCl2 channel functions as a histamine-gated chloride channel (Gisselmann et al., 2002; Pantazis et al., 2008), slow repolarization of LMCs in Lai-GAL4/UAS-TeTxLC flies could simply reflect defective histamine recycling, which subsequently causes prolonged opening of $\mathrm{HisCl} 2$ channels. Our intracellular recordings reveal normal amplitude $\left(w^{1118}\right.$ : amplitude $=5.98 \pm 0.60 \mathrm{mV}$, vs Lai-Gal4/UAS-TeTxLC: amplitude $=6.14 \pm 0.51 \mathrm{mV}, t_{(15)}=0.20$, $p=0.92$, two-tailed Student's $t$ test; Fig. $2 C$ ) and rise time in L1 and L2 neurons in Lai-GAL4/UAS-TeTxLC flies $\left(w^{1118}\right.$ : rise time $=0.079 \pm 0.0046 \mathrm{~s}$, vs Lai-Gal4/UAS-TeTxLC: rise time $=0.091 \pm 0.0028 \mathrm{~s}, t_{(15)}=2.01, \quad p=0.063$, two-tailed Student's $t$ test; Fig. $2 C$ ), reflecting normal histamine release from photoreceptor cells and normal opening of $\mathrm{HisCl} 2$ channels in L1 and L2 neurons. Released histamine is taken up by lamina glia rapidly and conjugated to $\beta$-alanine to form inactive carcinine in epithelial glia (Borycz et al., 2002; Richardt et al., 2003; Edwards and Meinertzhagen, 2010). Next, we examined the distribution of histamine by labeling the cryosections of the fly head with antihistamine antibodies. The Lai-GAL4/UAS-TeTxLC flies show normal distribution of histamine in the retina and the lamina. The relative signaling for histamine in the lamina over the retina in Lai- 
A

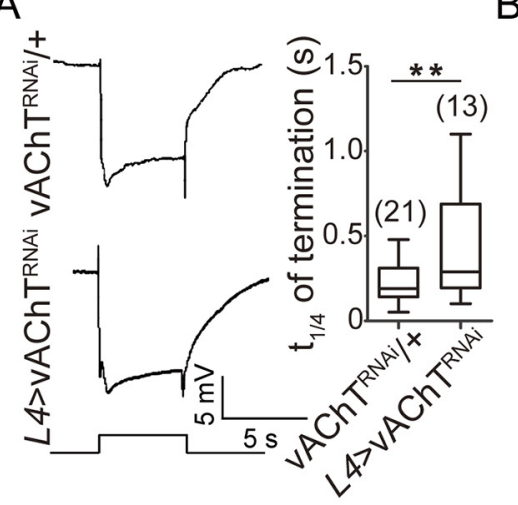

B

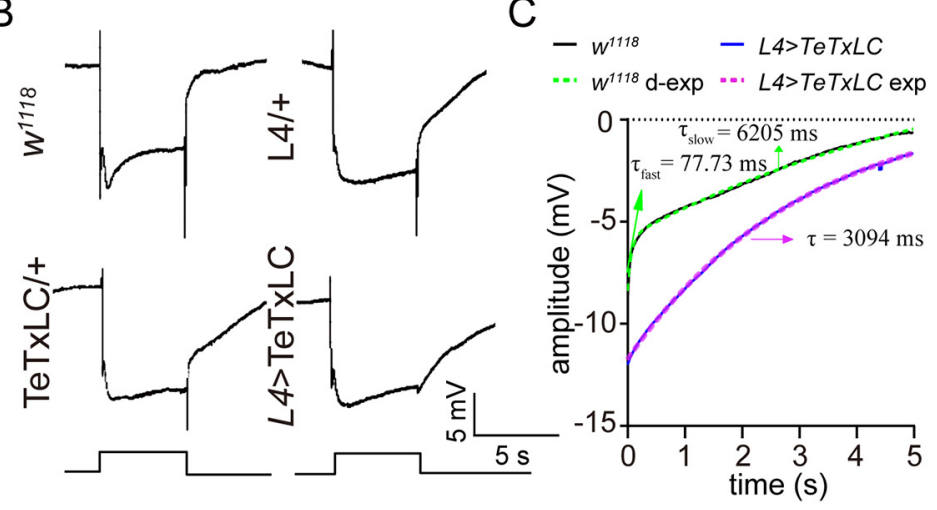

$\mathrm{D}$

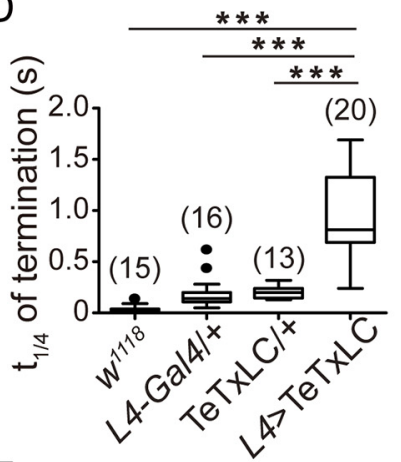

F

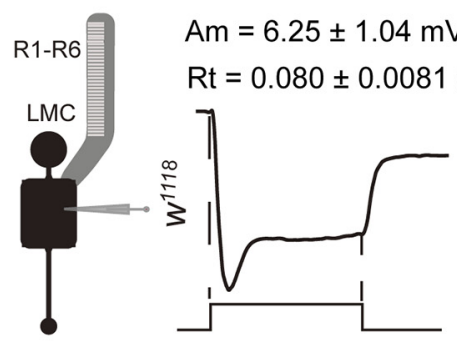

$\mathrm{H}$

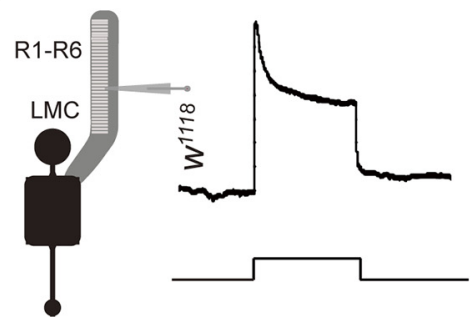

E
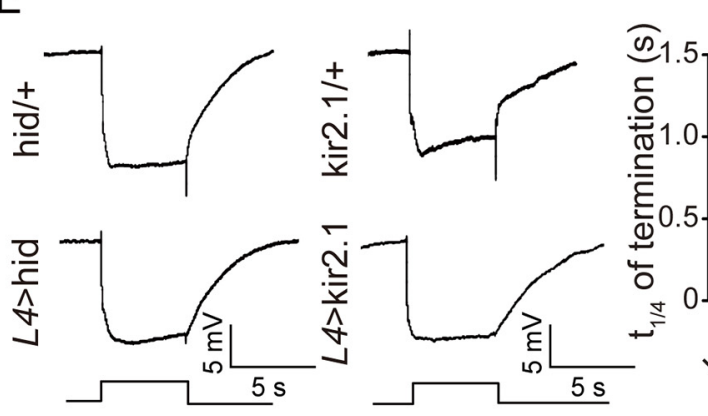

(13)

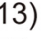

*** (22)

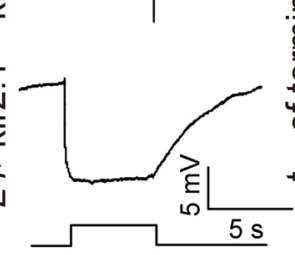

G

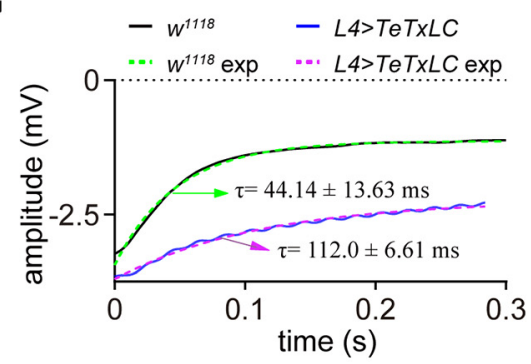

I

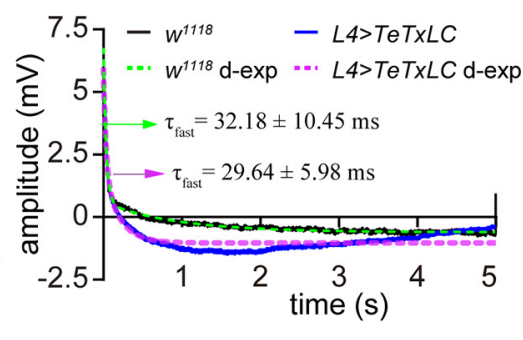

Figure 4. Blocking synaptic $A C h$ release from $L 4$ neurons leads to slow repolarization in $L M C s$. A, ERG recording traces of $L 4-G A L 4 / U A S-v A C h T^{R N A i}$ and UAS-vAChT $T^{R N A i} /+$ flies. Right, Quantification of the time $\left(\mathrm{t}_{1 / 4}\right)$ required for a $1 / 4$ recovery and the number of recorded flies. $\boldsymbol{B}$, ERG recording traces of the flies with expression of TeTxLC using L4-GAL4 driver. Event markers represent $5 \mathrm{~s}$ orange light pulses. Calibration: ERG traces throughout all figures, $5 \mathrm{mV}$. C, The decay of ERG recordings in WT and L4-Gal4/UAS-TeTxLC flies is fit with double or single exponential decay fit, respectively. Solid lines indicate the averaged experimental ERG recording decay traces. Dashed lines indicate the exponential decay fit. $w^{1118}: R^{2}=99.45 \% ;$ L4-Gal4/UAS-TeTxLC: $R^{2}=99.72 \%$. Arrows indicate decay constants for the fast and slow component of the exponential. $\boldsymbol{D}, Q$ Quantification of the time $\left(\mathrm{t}_{1 / 4}\right)$ required for a $1 / 4$ recovery from the responses on stimulation cessation and the number of recorded flies for each genotype. $\boldsymbol{E}$, ERG recording traces of the flies with expression of hid, or Kir2.1 using L4-GAL4 driver. Event markers represent $5 \mathrm{~s}$ orange light pulses. Calibration: ERG traces throughout all figures, $5 \mathrm{mV}$. Right, Quantification of the time $\left(\mathrm{t}_{1 / 4}\right)$ required for a $1 / 4$ recovery from the responses on stimulation cessation and the number of recorded flies for each genotype. $\boldsymbol{F}$, Intracellular recording represents slow repolarization in L4-GAL4/UAS-TeTxLC LMCs. Event markers represent $200 \mathrm{~ms}$ light pulses. Calibration: intracellular recording traces, $2.5 \mathrm{mV}$. Top, Measurements of the amplitude $(\mathrm{Am})$ and rise time $(\mathrm{Rt})$ of light-induced hyperpolarization. $\mathbf{G}$, The decay of intracellular recordings in LMCs are fit with single exponential decay fit. Solid lines indicate the averaged decay trace of experimental intracellular recording. Dashed lines indicate the single exponential decay fit. $w^{1118}: R^{2}=98.72 \% ; L 4-G a / 4 / U A S-T e T x L C: R^{2}=98.52 \%$. Arrows indicate decay constants for the exponential fit. $\boldsymbol{H}$, Intracellular recording reveals normal light response in $w^{1118}$ and L4-GAL4/UAS-TeTXLC photoreceptors. Event markers represent $5 \mathrm{~s}$ orange light pulses. Calibration: intracellular recording traces, $5 \mathrm{mV}$. $I$, The decay of intracellular recordings in photoreceptor cells are fit with double exponential decay fit. Solid lines indicate the averaged decay trace of experimental intracellular recording. Dashed lines indicate the exponential decay fit. $W^{1118}: R^{2}=91.43 \%$; $L 4-G a l 4 / U A S-$ TeTxLC: $R^{2}=73.21 \%$. Arrows indicate decay constants for the fast component of the exponential fit. $* * p<0.01 . * * * p<0.001$. 
GAL4/UAS-TeTxLC flies is comparable to that of the control flies $(2.89 \pm 0.38$ vs $2.4 \pm 0.23, t_{(9)}=1.19, p=0.26$, two-tailed Student's $t$ test; Fig. $3 A$ ). These results indicate that blocking synaptic output from ACs does not affect histamine recycling.

Previous studies have shown that applying the neurotransmitter GABA or ACh induces depolarization of LMCs (Hardie, 1987). Thus, we examined whether GABA or ACh signaling facilitates light off-induced LMC neuron repolarization. We find that knockdown of either AChR $\alpha 5$ or $\alpha 7$ subunit in L2 neurons results in slow ERG termination $\left(U A S-n A C h R \alpha 5^{R N A i} /+: \mathrm{t}_{1 / 4}=0.15 \pm\right.$ $0.027 \mathrm{~s}$, vs L2-Gal4/UAS-nAChR $\alpha 5^{R N A i}: \mathrm{t}_{1 / 4}=$ $0.39 \pm 0.067 \mathrm{~s}, \quad t_{(32)}=3.47, p=0.0015 ; \quad U A S-$ $n A C h R \alpha 7^{R N A i} /+: \mathrm{t}_{1 / 4}=0.17 \pm 0.036 \mathrm{~s}$, vs L2Gal4/UAS-nAChR $\alpha 7^{R N A i} \mathrm{t}_{1 / 4}=0.31 \pm 0.029 \mathrm{~s}$, $t_{(26)}=3.04, p=0.0045$, two-tailed Student's $t$ test; Fig. $3 B, C)$. In contrast, knockdown of other AChR subunits or GABA receptors in L2 neurons exhibits normal ERG termination (Fig. 3C,D). These results demonstrate that L2 neurons receive $\mathrm{ACh}$ signaling to facilitate light off-induced L1 and $\mathrm{L} 2$ neuron rapid repolarization.

ACs are lamina intrinsic multicolumnar neurons, and the arbors of each AC cell span multiple columns (Tuthill et al., 2013). The reconstruction data from serial electron microscopy indicate that ACs form a synaptic interaction with L1 and L2 neurons (Rivera-Alba et al., 2011). We use the GRASP technique to further verify the synaptic interaction between ACs and L1/L2 neurons (Feinberg et al., 2008; Guo et al., 2019). GFP1-10 is expressed in ACs, and GFP11 is expressed in L2 neurons; and then columnar signals of reconstituted GFP are observed in the lamina region (Fig. $3 E$ ). In contrast, no reconstituted GFP signals are observed in the control flies (Fig. $3 E$ ). These results provide further evidence supporting earlier findings.

\section{Synaptic ACh signaling from L4 neurons facilitates light off-induced repolarization in $\mathrm{L} 2$ neurons}

Given that L2 neurons also directly receive synaptic ACh signaling from L4 neurons (Takemura et al., 2011), we investigated whether synaptic ACh signaling from L4 neurons also facilitates light offinduced L2 repolarization and ERG termination. Interestingly, knockdown of vAChT in L4 neurons leads to the slow ERG termination phenotype $\left(U A S-v A C h T^{R N A i} /+: \mathrm{t}_{1 / 4}=0.23 \pm 0.026 \mathrm{~s}\right.$, vs $L 4-G a l 4 / U A S-v A C h T^{R N A i}, \mathrm{t}_{1 / 4}=0.45 \pm 0.085 \mathrm{~s}, t_{(32)}=3.14$, $p=0.0037$, two-tailed Student's $t$ test; Fig. $4 A$ ). Convincingly, blocking the neurotransmitter release from L4 neurons via ectopic expression of TeTxLC results in slow ERG termination, and a satisfied single exponential decay fit with $\tau=3094 \mathrm{~ms}$ is obtained for the decay of ERG recording in these flies (Fig. 4B, $C$ ), indicating that the regulation for the fast decay of ERG recording is also disrupted in L4-Gal4/UAS-TeTxLC flies. Consistently, blocking the neurotransmitter release from L4 neurons through different genetic approaches also results in slow ERG termination $\left(w^{1118}: \mathrm{t}_{1 / 4}=0.035 \pm 0.011 \mathrm{~s}\right.$, vs L4-Gal4/UASTeTxLC: $\mathrm{t}_{1 / 4}=0.94 \pm 0.096 \mathrm{~s}, t_{(33)}=8.17, p<0.001 ;$ L4-Gal4/+: $\mathrm{t}_{1 / 4}=0.19 \pm 0.037 \mathrm{~s}$, vs L4-Gal4/UAS-TeTxLC: $\mathrm{t}_{1 / 4}=0.94 \pm 0.096 \mathrm{~s}$, $t_{(34)}=6.71, p<0.001 ;$ UAS-TeTxLC/+: $\mathrm{t}_{1 / 4}=0.21 \pm 0.016 \mathrm{~s}$, vs L4Gal4/UAS-TeTxLC: $\mathrm{t}_{1 / 4}=0.94 \pm 0.096 \mathrm{~s}, t_{(31)}=6.14, p<0.001$; UAS- hid/+: $\mathrm{t}_{1 / 4}=0.24 \pm 0.019$ s, vs L4-Gal4/UAS-hid: $\mathrm{t}_{1 / 4}=$ $0.61 \pm 0.091 \mathrm{~s}, t_{(23)}=3.8, p<0.001 ;$ UAS-Kir2.1/+: $\mathrm{t}_{1 / 4}=0.18 \pm$
0.03 s, vs L4-Gal4/UAS-Kir2.1: $\mathrm{t}_{1 / 4}=0.65 \pm 0.06 \mathrm{~s}, t_{(34)}=6.04$, $p<0.001$, two-tailed Student's $t$ test; Fig. $4 D, E)$. These data show that blocking synaptic ACh release from L4 neurons either by disruption of synaptic release molecular components or genetically induced hyperpolarization that indirectly blocks synaptic release leads to slow ERG termination.

To confirm synaptic ACh signaling from L4 neurons is also required for rapid repolarization of $\mathrm{L} 2$ neurons, we conducted intracellular recordings in L1 and L2 neurons. Compared with control flies, turning the light off induces slow repolarization of L1 and L2 neurons in L4-Gal4/UAS-TeTxLC flies ( $w^{1118}$ : single exponential decay fit, $\tau=44.14 \pm 13.63 \mathrm{~ms}$, vs $L 4$-Gal4/UASTeTxLC: single exponential decay fit, $\tau=112.0 \pm 6.61 \mathrm{~ms}$, $t_{(4)}=4.48, p=0.011$, two-tailed Student's $t$ test; Fig. $\left.4 F, G\right)$. In contrast, intracellular recordings in the photoreceptors revealed normal fast decay in the photoreceptors of L4-Gal4/UASTeTXLC flies ( $w^{1118}$ : double exponential decay fit, $\tau_{\text {fast }}=$ $32.18 \pm 10.45 \mathrm{~ms}$, vs L4-Gal4/UAS-TeTxLC: double exponential decay fit, $\tau_{\text {fast }}=29.64 \pm 5.98 \mathrm{~ms}, t_{(4)}=0.21, p=0.84$, two-tailed Student's $t$ test; Fig. $4 H, I$ ). These data demonstrate that synaptic ACh signaling from L4 neurons also facilitates light off-induced rapid repolarization of L1 and L2 neurons.

\section{Synaptic ACh signaling from both ACs and L4 neurons facilitate ERG termination simultaneously}

Given that blocking synaptic ACh signaling from either ACs or L4 neurons results in slow repolarization of L2 neurons, we investigated whether L2 neurons receive synaptic ACh signaling from ACs and L4 neurons simultaneously or successively. We blocked neurotransmitter release from both ACs and L4 neurons via ectopic expression of TeTxLC. We found that Lai-GAL4;L4Gal4/UAS-TeTxLC flies exhibit slower ERG termination than either Lai-GAL4/UAS-TeTxLC or L4-Gal4/UAS-TeTxLC flies (UAS-TeTxLC/+: $\mathrm{t}_{1 / 4}=0.16 \pm 0.037 \mathrm{~s}$, vs Lai-Gal4;L4-Gal4/ UAS-TeTxLC: $\mathrm{t}_{1 / 4}=1.4 \pm 0.12 \mathrm{~s}, \mathrm{t}_{(29)}=7.89, p<0.0001$; LaiGal4/UAS-TeTxLC: $\mathrm{t}_{1 / 4}=1.06 \pm 0.053$ s, vs Lai-Gal4;L4-Gal4/ UAS-TeTxLC: $\mathrm{t}_{1 / 4}=1.4 \pm 0.12 \mathrm{~s}, t_{(38)}=2.6, p=0.013 ;$ L4-Gal4/ UAS-TeTxLC: $\mathrm{t}_{1 / 4}=1.04 \pm 0.075$ s, vs Lai-Gal4;L4-Gal4/UASTeTxLC: $\mathrm{t}_{1 / 4}=1.4 \pm 0.12 \mathrm{~s}, t_{(40)}=2.59, p=0.013$, two-tailed Student's $t$ test; Fig. $5 A, B)$. These results indicate that ACh signaling from both ACs and L4 neurons is essential for the rapid ERG termination, and suggests that this ACh signaling might simultaneously facilitate ERG termination. 
A
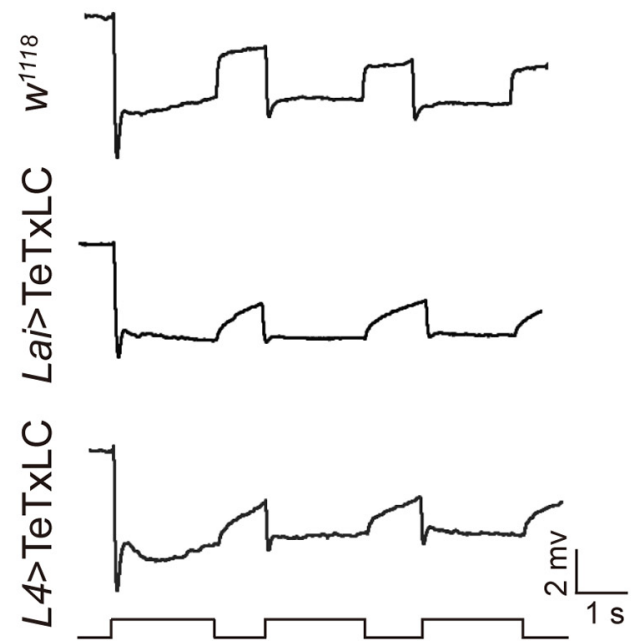

C

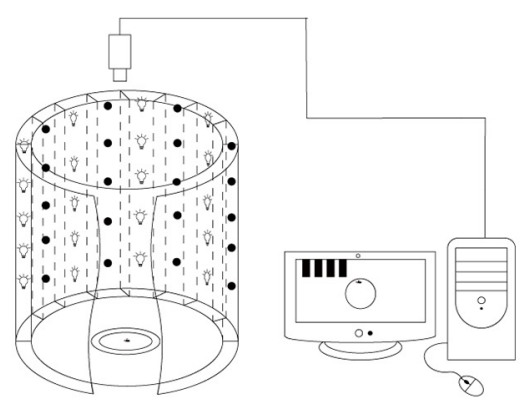

B

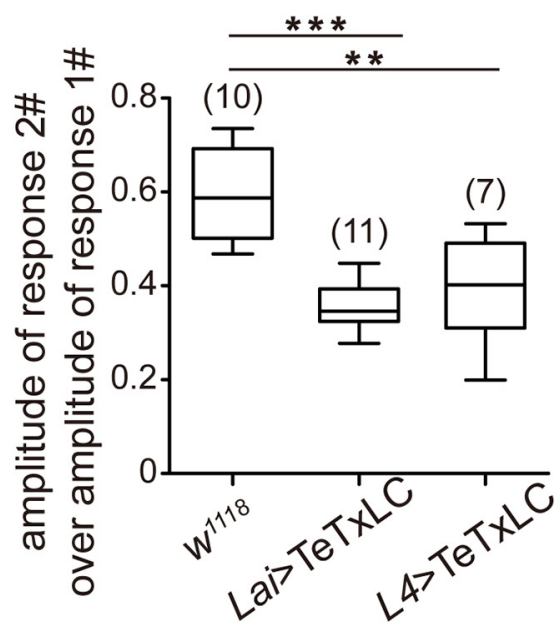

D

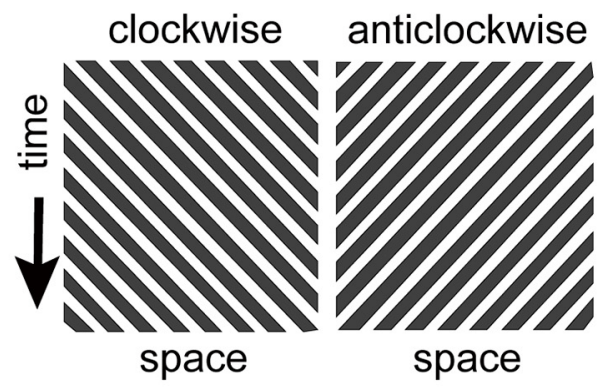

E

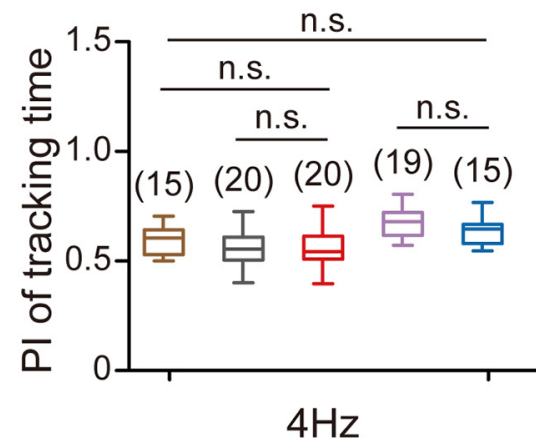

- Lai>TeTXLC

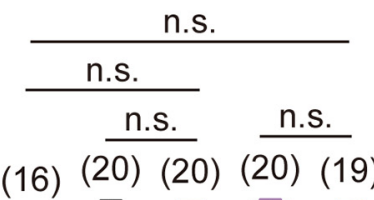

(16) (20) (20) (20) (19)

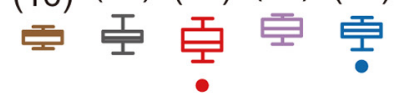

$L 4 /+$ L4>TeTXLC

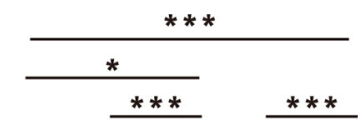

(16) $(20)$

(20)

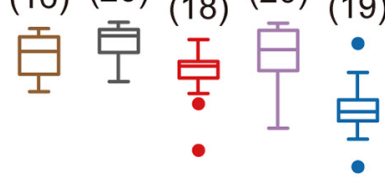

Figure 6. Parallel ACh signaling facilitates LMCs repolarization and the optomotor response of the flies in high-frequency light stimulation. $A$, Intracellular recordings represent the light response in LMCs for each genotype. Fly eyes were stimulated with a series of $2 \mathrm{~s}$ light pulses with a $1 \mathrm{~s}$ dark interval. Event markers represent $1 \mathrm{~s}$ light pulses. Calibration: intracellular recording traces, $2 \mathrm{mV}$. $\boldsymbol{B}$, Boxplots with whiskers represent the amplitudes of light response 2\# over the amplitude of light response $1 \#$ for each genotype and condition (with maximum 1.5 interquartile ranges). The number of recorded flies for each genotype are presented. C, Schematic representation of the experimental apparatus. Only half of the LED is displayed. $\boldsymbol{D}$, Full-field stimuli used in all behavioral experiments. The space-time diagrams represent the luminance patterns displayed to the fly in the arena. $\boldsymbol{E}$, Boxplots with whiskers represent the performance index of tracking time for each genotype and condition (with maximum 1.5 interquartile ranges). The number of flies for each genotype and condition are presented. $* p<0.05 . * * p<0.01$. $* * * p<0.001$; n.S., Not significant $(p>0.05)$.

\section{Synaptic ACh signaling from either ACs or L4 neurons is essential for visual behavior}

Rapid repolarization of LMCs has particular importance in visual signaling, which must be reset rapidly to respond to subsequent stimuli. To investigate the physiological roles of synaptic ACh signaling from either ACs or L4 neurons in L1 and L2 neurons, we performed intracellular recordings to examine the responses of L1 and L2 neurons to a series of $2 \mathrm{~s}$ light pulses with a $1 \mathrm{~s}$ dark interval. The L1 and L2 neurons of WT flies show rapid repolarization when the light is off, and subsequent light stimulation evokes a comparable hyperpolarization $\left(w^{1118}: 0.6 \pm 0.031\right.$, vs Lai-Gal4/UAS-TeTxLC: $0.36 \pm 0.014, t_{(19)}=7.3, \quad p<0.001$; $w^{1118}: \quad 0.6 \pm 0.031$, vs L4-Gal4/UAS-TeTxLC: $0.4 \pm 0.043$, $t_{(15)}=3.89, p=0.0014$, two-tailed Student's $t$ test; Fig. $\left.6 A, B\right)$. In contrast, L1 and L2 neurons of either Lai-GAL4/UAS-TeTxLC or L4-Gal4/UAS-TeTxLC flies show slow repolarization when the light is off, and subsequent light stimulation induces reduced hyperpolarization (Fig. $6 A, B$ ). These observations indicate that 
synaptic ACh signaling from either ACs or L4 neurons to L2 neurons facilitates L1 and L2 neurons rapid repolarization to respond to subsequent stimuli.

To investigate the potential functional role of synaptic ACh signaling from either ACs or L4 neurons to L2 neurons in visual behavior, we assessed the optomotor responses of flies under various frequency light stimulations. We placed a single fly on a circular platform and examined its ability to track moving light patterns (Fig. 6C,D). With low-frequency moving light patterns (4 or $10 \mathrm{~Hz}$ ), LaiGAL4/UAS-TeTxLC and L4-Gal4/UASTeTxLC flies exhibit an ability to track moving patterns that is comparable to that of control flies (Fig. 6E). However, with high-frequency moving light patterns $(25 \mathrm{~Hz})$, both Lai-GAL4/UAS-TeTxLC and L4-Gal4/UAS-TeTxLC flies are less able to track moving patterns (UAS-TeTxLC/+: PITT $=0.85 \pm 0.024$ vs Lai-Gal4/UAS-TeTxLC: PITT $=0.76 \pm 0.027, t_{(32)}=2.24, p=0.032$; Lai-Gal4/+: $\quad$ PITT $=0.91 \pm 0.016$, vs Lai-Gal4/UAS-TeTxLC: PITT $=0.76 \pm 0.027, \quad t_{(36)}=4.77, \quad p<0.001 ; \quad$ UAS-TeTxLC/ + , PITT $=0.85 \pm 0.024$, vs $L 4-G a l 4 / U A S-T e T x L C$, PITT $=0.6 \pm 0.027$, $t_{(33)}=6.68, p<0.001 ;$ L4-Gal4/+: PITT $=0.85 \pm 0.029$, vs L4-Gal4/ $U A S-T e T x L C$, PITT $=0.6 \pm 0.027, t_{(37)}=6.19, p<0.001$, unpaired $t$ tests; Fig. $6 E$ ). These findings demonstrate that synaptic ACh signaling from either ACs or L4 neurons to L2 neurons is essential for the optomotor response of flies in high-frequency light stimulation.

Based on the aforementioned data, two parallel neural circuits that facilitate L1 and L2 neurons repolarization are outlined. Light stimulation induces histamine release from photoreceptor axons to the extracellular space, which hyperpolarizes L1-L3 neurons and ACs via opening HisCl2 channels (Gengs et al., 2002; Y. Zheng et al., 2002; Pantazis et al., 2008). Light off triggers ACh release from both ACs and L4 neurons, which repolarizes L1 and L2 neurons via AChRs (Fig. 7).

\section{Discussion}

Our study identifies two parallel neural circuits that facilitate L1 and L2 neuron repolarization after light stimulation is turned off. Moreover, our work demonstrates that synaptic ACh signaling from either ACs or L4 neurons to L2 neurons is essential for the optomotor response of the flies in high-frequency light stimulation.

\section{ACh signaling facilitates LMCs repolarization and rapid ERG termination}

Abnormal ERGs frequently reflect deficits in photoreceptors as well as in retrograde signaling to photoreceptors. However, our previous study show that blocking synaptic output of ACs results in slow ERG termination (Hu et al., 2015). In this study, our intracellular recordings reveal normal termination in the photoreceptors, but slow repolarization in the L1 and L2 neurons in Lai-GAL4/UASTeTxLC flies. Moreover, we show that expression of Kir2.1 channel in either L1 or L2 neurons is able to induce slow ERG termination. These data demonstrate that slow repolarization of L1 and L2 neurons leads to slow ERG termination.

Although the molecular and cellular mechanisms of photoresponse termination in photoreceptors have been extensively investigated, the underlying circuit-based mechanism that regulates rapid termination of light response in LMCs is less studied. Light stimulation induces the inhibitory neurotransmitter histamine released from photoreceptors to hyperpolarize postsynaptic L1-L3 and ACs by opening their HisCl2 channels (Huguenard, 1996; Gisselmann et al., 2002; Pantazis et al., 2008; Hu et al., 2015). Intracellular recording and voltage imaging in L2 neurons have shown that light flashes evoke transient hyperpolarization in L2 neurons that rapidly returns to the dark resting level (Pantazis et al., 2008; Yang et al., 2016). In our previous study, both L1 and L2 neurons express $\mathrm{I}_{\mathrm{h}}$ channels, which are normally activated at negative potentials $<-50 \mathrm{mV}$ (Biel et al., 2009; Hu et al., 2015). Thus, it is reasonable that histamine-induced hyperpolarization activates $\mathrm{I}_{\mathrm{h}}$ channels in L1 and L2 neurons to return their membrane potential to the resting level. However, previous intracellular recordings in L1 and L2 neurons have shown that the membrane potential cannot reach the resting level on longlasting light stimulation (L. Zheng et al., 2006). Furthermore, voltage imaging in L2 neurons reveals that a dark flash induces a transient depolarization in L2 neurons (Yang et al., 2016). These results indicate that L2 neurons may receive some excitatory signaling to promote rapid repolarization during dark flash or light off conditions. Another earlier study find that applying either GABA or ACh onto LMCs induces depolarization of LMCs (Hardie, 1987). In this study, we show that either blocking synaptic ACh signaling or depletion of AChRs in L2 neurons results in slow repolarization of L1 and L2 neurons. However, depletion of GABA receptors from $\mathrm{L} 2$ neurons fails to induce slow repolarization of L1 and L2 neurons. Moreover, we show that knockdown of either Drosophila nAChR- $\alpha 5$ (D $\alpha 5)$ or nAChR- $\alpha 7$ (D $\alpha 7)$ subunit in L2 neurons results in slow ERG termination. nAChRs mediate fast excitatory synaptic transmission on binding by ACh. Ten $\mathrm{nAChR}$ subunits have been identified in fly genome, and three of them $(\mathrm{D} \alpha 5, \mathrm{D} \alpha 6$, and $\mathrm{D} \alpha 7)$ are more homologous to vertebrate $\alpha 7$ receptors than to other insect nicotinic receptors (Littleton and Ganetzky, 2000; Grauso et al., 2002). These receptors have unique properties that can form homomeric channels consisting of only the $\alpha$ subunit, and have high permeability to calcium and rapid desensitization (Couturier et al., 1990; Seguela et al., 1993). These results provide the first evidence that ACh signaling facilitates rapid repolarization of L1 and L2 neurons. 


\section{Parallel synaptic ACh signaling from both ACs and L4 neurons regulates fly visual behavior}

Vertebrates and insects share many structural, functional, and developmental features in their visual systems. The synaptic signaling from horizontal cells to bipolar cells contributes to many visual functions, such as shaping receptive field and light adaptation (Dowling, 1991; Thoreson and Mangel, 2012). The ACs in the fly visual system, which are structurally equal to horizontal cells in vertebrates, receive synaptic input from photoreceptors and project onto T1 neurons, photoreceptor cells, LMCs, and epithelial glia (Rivera-Alba et al., 2011). Previous immunocytochemistry studies have revealed that ACs are both glutamatergic and cholinergic neurons (Yasuyama et al., 1996; Kolodziejczyk et al., 2008). ACs are hyperpolarized on light stimulation, whereas they are activated to release both glutamate and ACh when lights are turned off (Huguenard, 1996; Gisselmann et al., 2002; Pantazis et al., 2008; Hu et al., 2015). Our previous study demonstrates that feedback glutamate signaling from ACs to photoreceptors helps the fly maintain light sensitivity in the presence of ambient light. In this study, we show that synaptic ACh signaling from ACs to L2 neurons is essential for rapid repolarization of L1 and L2 neurons and the optomotor response of the fly during high-frequency light stimulation. These studies illustrate that ACs use different signaling to achieve their abilities for high light sensitivity and high temporal resolution (Montell, 1999; Tuthill et al., 2013; Hu et al., 2015). In addition, ACs also play critical roles in maintaining the response of the fly to regressive rotation stimulations as well as high-contrast and low-contrast stimulations (Tuthill et al., 2013).

Previous observations on Drosophila and the housefly Musca have shown that L2 and L4 neurons form reciprocal connections (Strausfeld and Campos-Ortega, 1973; Takemura et al., 2011). Immunocytochemistry studies have determined that L4 neurons are also cholinergic neurons (Yasuyama et al., 1996; Kolodziejczyk et al., 2008). These morphologic and immunocytochemistry evidence support the idea that L2 neurons might receive synaptic ACh signaling from L4 neurons to facilitate L1 and $\mathrm{L} 2$ repolarization. In this study, we show that blocking synaptic ACh signaling from L4 neurons also results in slow repolarization of L1 and L2 neurons and reduces the optomotor response of the flies in high-frequency light stimulation conditions. Light off-triggered activation signaling in L4 neurons needs to be further investigated because L4 neurons do not receive direct input from photoreceptors.

In this study, we show that $\mathrm{L} 2$ neurons receive synaptic $\mathrm{ACh}$ signaling simultaneously from both ACs and L4 neurons. The inputs from ACs and L4 neurons may encode additionally information for image-forming visual processing. The fly optic lobes are assembled into retinotopic columns and each corresponding to a small region of visual space. L2 and L4 neurons form reciprocal connection in the same cartridge so that they can communicate local vision signal from each other (Meinertzhagen and O'Neil, 1991; Takemura et al., 2011). Consistent with the structure properties, electrically silenced either L2 or ACs leads to reduced ability in low contrast rotation, stripe oscillation, progressive rotation, as well as OFF motion (Tuthill et al., 2013). In contrast, the multicolumnar AC cells are lamina intrinsic neurons with a rich connectivity structure that both receive and send synapses along the length of a cartridge (Rivera-Alba et al., 2011). The anatomy properties of AC cells suggest that they function as feedback neurons and integrate the image-forming visual information from multicolumn. Electrically silenced either L2 neurons or AC cells results in increased ability in contrast nulling (Tuthill et al., 2013). Given that L1 and L2 neurons receive multiple inputs, including $\mathrm{L} 4, \mathrm{ACs}, \mathrm{C} 2, \mathrm{C} 3$, and Lawf neurons (Joesch et al., 2010; Rivera-Alba et al., 2011), the potential role of other neurons in L1 and L2 neuron repolarization also needs to be investigated further.

\section{References}

Agee HR, Park ML (1975) Use of the electroretinogram to measure the quality of vision of the fruit fly. Environ Lett 10:171-176.

Baik LS, Au DD, Nave C, Foden AJ, Enrriquez-Villalva WK, Holmes TC (2019) Distinct mechanisms of Drosophila CRYPTOCHROME-mediated light-evoked membrane depolarization and in vivo clock resetting. Proc Natl Acad Sci USA 116:23339-23344.

Baines RA, Uhler JP, Thompson A, Sweeney ST, Bate M (2001) Altered electrical properties in Drosophila neurons developing without synaptic transmission. J Neurosci 21:1523-1531.

Berson DM, Dunn FA, Takao M (2002) Phototransduction by retinal ganglion cells that set the circadian clock. Science 295:1070-1073.

Biel M, Wahl-Schott C, Michalakis S, Zong X (2009) Hyperpolarization-activated cation channels: from genes to function. Physiol Rev 89:847-885.

Borycz J, Borycz JA, Loubani M, Meinertzhagen IA (2002) tan and ebony genes regulate a novel pathway for transmitter metabolism at fly photoreceptor terminals. J Neurosci 22:10549-10557.

Bringmann A, Pannicke T, Grosche J, Francke M, Wiedemann P, Skatchkov SN, Osborne NN, Reichenbach A (2006) Muller cells in the healthy and diseased retina. Prog Retin Eye Res 25:397-424.

Burns ME, Baylor DA (2001) Activation, deactivation, and adaptation in vertebrate photoreceptor cells. Annu Rev Neurosci 24:779-805.

Cadetti L, Thoreson WB (2006) Feedback effects of horizontal cell membrane potential on cone calcium currents studied with simultaneous recordings. J Neurophysiol 95:1992-1995.

Chaturvedi R, Reddig K, Li HS (2014) Long-distance mechanism of neurotransmitter recycling mediated by glial network facilitates visual function in Drosophila. Proc Natl Acad Sci USA 111:2812-2817.

Couturier S, Bertrand D, Matter JM, Hernandez MC, Bertrand S, Millar N, Valera S, Barkas T, Ballivet M (1990) A neuronal nicotinic acetylcholine receptor subunit (alpha 7) is developmentally regulated and forms a homo-oligomeric channel blocked by alpha-BTX. Neuron 5:847-856.

Dowling JE (1991) Retinal neuromodulation: the role of dopamine. Vis Neurosci 7:87-97.

Edwards TN, Meinertzhagen IA (2010) The functional organisation of glia in the adult brain of Drosophila and other insects. Prog Neurobiol 90:471497.

Feinberg EH, Vanhoven MK, Bendesky A, Wang G, Fetter RD, Shen K, Bargmann CI (2008) GFP reconstitution across synaptic partners (GRASP) defines cell contacts and synapses in living nervous systems. Neuron 57:353-363.

Gengs C, Leung HT, Skingsley DR, Iovchev MI, Yin Z, Semenov EP, Burg MG, Hardie RC, Pak WL (2002) The target of Drosophila photoreceptor synaptic transmission is a histamine-gated chloride channel encoded by ort (hclA). J Biol Chem 277:42113-42120.

Gisselmann G, Pusch H, Hovemann BT, Hatt H (2002) Two cDNAs coding for histamine-gated ion channels in D. melanogaster. Nat Neurosci 5:1112.

Gordon MD, Scott K (2009) Motor control in a Drosophila taste circuit. Neuron 61:373-384.

Grauso M, Reenan RA, Culetto E, Sattelle DB (2002) Novel putative nicotinic acetylcholine receptor subunit genes, Dalpha5, Dalpha6 and Dalpha7, in Drosophila melanogaster identify a new and highly conserved target of adenosine deaminase acting on RNA-mediated A-to-I pre-mRNA editing. Genetics 160:1519-1533.

Guo C, Pan Y, Gong Z (2019) Recent advances in the genetic dissection of neural circuits in Drosophila. Neurosci Bull 35:1058-1072.

Han J, Reddig K, Li HS (2007) Prolonged G(q) activity triggers fly rhodopsin endocytosis and degradation, and reduces photoreceptor sensitivity. EMBO J 26:4966-4973.

Han J, Gong P, Reddig K, Mitra M, Guo P, Li HS (2006) The fly CAMTA transcription factor potentiates deactivation of rhodopsin, a G proteincoupled light receptor. Cell 127:847-858.

Hardie R (1987) Is histamine a neurotransmitter in insect photoreceptors? J Comp Physiol A Neuroethol Sens Neural Behav Physiol 161:201-213. 
Hardie RC (1989) A histamine-activated chloride channel involved in neurotransmission at a photoreceptor synapse. Nature 339:704-706.

Hardie RC, Raghu P (2001) Visual transduction in Drosophila. Nature 413:186-193.

Hardie RC, Raghu P, Moore S, Juusola M, Baines RA, Sweeney ST (2001) Calcium influx via TRP channels is required to maintain PIP2 levels in Drosophila photoreceptors. Neuron 30:149-159.

Hattar S, Liao HW, Takao M, Berson DM, Yau KW (2002) Melanopsin-containing retinal ganglion cells: architecture, projections, and intrinsic photosensitivity. Science 295:1065-1070.

Heisenberg M (1971) Separation of receptor and lamina potentials in the electroretinogram of normal and mutant Drosophila. J Exp Biol 55:85100 .

Hu W, Wang T, Wang X, Han J (2015) Ih channels control feedback regulation from amacrine cells to photoreceptors. PLoS Biol 13:e1002115.

Hu W, Wan D, Yu X, Cao J, Guo P, Li HS, Han J (2012) Protein Gq modulates termination of phototransduction and prevents retinal degeneration. J Biol Chem 287:13911-13918.

Huguenard JR (1996) Low-threshold calcium currents in central nervous system neurons. Annu Rev Physiol 58:329-348.

Joesch M, Schnell B, Raghu SV, Reiff DF, Borst A (2010) ON and OFF pathways in Drosophila motion vision. Nature 468:300-304

Kitamoto T (2001) Conditional modification of behavior in Drosophila by targeted expression of a temperature-sensitive shibire allele in defined neurons. J Neurobiol 47:81-92.

Kolodziejczyk A, Sun X, Meinertzhagen IA, Nassel DR (2008) Glutamate, GABA and acetylcholine signaling components in the lamina of the Drosophila visual system. PLoS One 3:e2110.

Laitko U, Hofmann KP (1998) A model for the recovery kinetics of rod phototransduction, based on the enzymatic deactivation of rhodopsin. Biophys J 74:803-815.

Lee T, Luo L (1999) Mosaic analysis with a repressible cell marker for studies of gene function in neuronal morphogenesis. Neuron 22:451-461.

Littleton JT, Ganetzky B (2000) Ion channels and synaptic organization: analysis of the Drosophila genome. Neuron 26:35-43.

Luan H, Lemon WC, Peabody NC, Pohl JB, Zelensky PK, Wang D, Nitabach MN, Holmes TC, White BH (2006) Functional dissection of a neuronal network required for cuticle tanning and wing expansion in Drosophila. J Neurosci 26:573-584

Macpherson LJ, Zaharieva EE, Kearney PJ, Alpert MH, Lin TY, Turan Z, Lee CH, Gallio M (2015) Dynamic labelling of neural connections in multiple colours by trans-synaptic fluorescence complementation. Nat Commun 6:10024.

McNabb SL, Baker JD, Agapite J, Steller H, Riddiford LM, Truman JW (1997) Disruption of a behavioral sequence by targeted death of peptidergic neurons in Drosophila. Neuron 19:813-823.

Meinertzhagen IA, O’Neil SD (1991) Synaptic organization of columnar elements in the lamina of the wild type in Drosophila melanogaster. J Comp Neurol 305:232-263.

Montell C (1999) Visual transduction in Drosophila. Annu Rev Cell Dev Biol 15:231-268.

Ni JD, Baik LS, Holmes TC, Montell C (2017) A rhodopsin in the brain functions in circadian photoentrainment in Drosophila. Nature 545:340-344.

Nitabach MN, Wu Y, Sheeba V, Lemon WC, Strumbos J, Zelensky PK, White BH, Holmes TC (2006) Electrical hyperexcitation of lateral ventral pacemaker neurons desynchronizes downstream circadian oscillators in the fly circadian circuit and induces multiple behavioral periods. J Neurosci 26:479-489.

Pantazis A, Segaran A, Liu CH, Nikolaev A, Rister J, Thum AS, Roeder T, Semenov E, Juusola M, Hardie RC (2008) Distinct roles for two histamine receptors (hclA and hclB) at the Drosophila photoreceptor synapse. J Neurosci 28:7250-7259.

Pecot MY, Tadros W, Nern A, Bader M, Chen Y, Zipursky SL (2013) Multiple interactions control synaptic layer specificity in the Drosophila visual system. Neuron 77:299-310.
Pfeiffer BD, Jenett A, Hammonds AS, Ngo TT, Misra S, Murphy C, Scully A, Carlson JW, Wan KH, Laverty TR, Mungall C, Svirskas R, Kadonaga JT, Doe CQ, Eisen MB, Celniker SE, Rubin GM (2008) Tools for neuroanatomy and neurogenetics in Drosophila. Proc Natl Acad Sci USA 105:9715-9720.

Pfeiffer BD, Ngo TT, Hibbard KL, Murphy C, Jenett A, Truman JW, Rubin GM (2010) Refinement of tools for targeted gene expression in Drosophila. Genetics 186:735-755.

Rajaram S, Scott RL, Nash HA (2005) Retrograde signaling from the brain to the retina modulates the termination of the light response in Drosophila. Proc Natl Acad Sci USA 102:17840-17845.

Richardt A, Kemme T, Wagner S, Schwarzer D, Marahiel MA, Hovemann BT (2003) Ebony, a novel nonribosomal peptide synthetase for beta-alanine conjugation with biogenic amines in Drosophila. J Biol Chem 278:41160-41166.

Rister J, Pauls D, Schnell B, Ting CY, Lee CH, Sinakevitch I, Morante J, Strausfeld NJ, Ito K, Heisenberg M (2007) Dissection of the peripheral motion channel in the visual system of Drosophila melanogaster. Neuron 56:155-170.

Rivera-Alba M, Vitaladevuni SN, Mishchenko Y, Lu Z, Takemura SY, Scheffer L, Meinertzhagen IA, Chklovskii DB, de Polavieja GG (2011) Wiring economy and volume exclusion determine neuronal placement in the Drosophila brain. Curr Biol 21:2000-2005.

Seguela P, Wadiche J, Dineley-Miller K, Dani JA, Patrick JW (1993) Molecular cloning, functional properties, and distribution of rat brain alpha 7: a nicotinic cation channel highly permeable to calcium. J Neurosci 13:596-604.

Strausfeld NJ, Campos-Ortega JA (1973) The L4 monopolar neurone: a substrate for lateral interaction in the visual system of the fly Musca domestica (L.). Brain Res 59:97-117.

Sweeney ST, Broadie K, Keane J, Niemann H, O’Kane CJ (1995) Targeted expression of tetanus toxin light chain in Drosophila specifically eliminates synaptic transmission and causes behavioral defects. Neuron 14:341-351.

Takemura SY, Karuppudurai T, Ting CY, Lu Z, Lee CH, Meinertzhagen IA (2011) Cholinergic circuits integrate neighboring visual signals in a Drosophila motion detection pathway. Curr Biol 21:2077-2084.

Thoreson WB, Mangel SC (2012) Lateral interactions in the outer retina. Prog Retin Eye Res 31:407-441.

Tian Y, Hu W, Tong H, Han J (2012) Phototransduction in Drosophila. Sci China Life Sci 55:27-34.

Tian Y, Li T, Sun M, Wan D, Li Q, Li P, Zhang ZC, Han J, Xie W (2013) Neurexin regulates visual function via mediating retinoid transport to promote rhodopsin maturation. Neuron 77:311-322.

Tuthill JC, Nern A, Holtz SL, Rubin GM, Reiser MB (2013) Contributions of the 12 neuron classes in the fly lamina to motion vision. Neuron 79:128140.

Wang T, Montell C (2007) Phototransduction and retinal degeneration in Drosophila. Pflugers Arch 454:821-847.

Yang HH, St-Pierre F, Sun X, Ding X, Lin MZ, Clandinin TR (2016) Subcellular imaging of voltage and calcium signals reveals neural processing in vivo. Cell 166:245-257.

Yasuyama K, Kitamoto T, Salvaterra PM (1996) Differential regulation of choline acetyltransferase expression in adult Drosophila melanogaster brain. J Neurobiol 30:205-218.

Zheng L, de Polavieja GG, Wolfram V, Asyali MH, Hardie RC, Juusola M (2006) Feedback network controls photoreceptor output at the layer of first visual synapses in Drosophila. J Gen Physiol 127:495-510.

Zheng Y, Hirschberg B, Yuan J, Wang AP, Hunt DC, Ludmerer SW, Schmatz DM, Cully DF (2002) Identification of two novel Drosophila melanogaster histamine-gated chloride channel subunits expressed in the eye. J Biol Chem 277:2000-2005.

Zhou L, Schnitzler A, Agapite J, Schwartz LM, Steller H, Nambu JR (1997) Cooperative functions of the reaper and head involution defective genes in the programmed cell death of Drosophila central nervous system midline cells. Proc Natl Acad Sci USA 94:5131-5136. 